Clemson University

TigerPrints

May 2020

\title{
Decomposition of Chemical Warfare Agent Simulants Utilizing Pyrolyzed Cotton Balls as Wicks
}

Bryan A. Lagasse

Clemson University, bryan.lagasse@gmail.com

Follow this and additional works at: https://tigerprints.clemson.edu/all_theses

\section{Recommended Citation}

Lagasse, Bryan A., "Decomposition of Chemical Warfare Agent Simulants Utilizing Pyrolyzed Cotton Balls as Wicks" (2020). All Theses. 3273.

https://tigerprints.clemson.edu/all_theses/3273

This Thesis is brought to you for free and open access by the Theses at TigerPrints. It has been accepted for inclusion in All Theses by an authorized administrator of TigerPrints. For more information, please contact kokeefe@clemson.edu. 


\section{DECOMPOSITION OF CHEMICAL WARFARE AGENT SIMULANTS UTILIZING PYROLYZED COTTON BALLS AS WICKS}

A Thesis
Presented to
the Graduate School of
Clemson University
In Partial Fulfillment
of the Requirements for the Degree
Master of Science
Chemistry
Bryan Alan Lagasse
May 2020
by
Accepted by:
Dr. Carlos Garcia, Committee Chair
Dr. Kenneth Marcus
Dr. Rakesh Sachdeva
Dr. Khitehead




\begin{abstract}
The thermal decomposition of chemical warfare agent simulants via a novel and simple approach is reported. Utilizing pyrolyzed cotton balls as a substrate for the delivery of an incendiary agent into a bulk volume of mustard and nerve agent simulants, significant enhancements in the burning rates were achieved. Under ambient conditions, this method was able to thermally decompose simulants for both HD (sulfur mustard) and GB (sarin) series chemical warfare agents at rates up to $3.1 \pm 0.4 \mathrm{~g} . \mathrm{min}^{-1}$ and $1.9 \pm 1.0 \mathrm{~g} . \mathrm{min}^{-1}$, respectively. The approach proposes a simple and more cost-effective way to decompose these dangerous substances under ambient conditions with the use of a wicking substrate rather than high temperature incineration equipment or chemical treatments.
\end{abstract}




\section{DEDICATION}

I would like to briefly thank those who have enabled me to achieve this goal. First to the Soldiers, leaders, and mentors without whom I would never have had the chance to pursue this opportunity. To my parents, who always supported me and gave me guidance and encouragement to keep struggling until I succeeded. Most importantly to my wife, Leah, who always listens to my crazy ramblings, keeps me focused, and provides me with the motivation to keep striving to improve. Finally, to my son, Mac, who doesn't realize yet how much his constant happy attitude and amazement in the world around him has helped me to stay grounded. 


\section{ACKNOWLEDGMENTS}

This manuscript is the culmination of months of work by myself, my advisor, and my committee: Dr. Carlos Garcia, Dr. Ken Marcus, Dr. Rakesh Sachdeva, and Dr. Dan Whitehead. I would like to acknowledge their immense contributions towards making this possible by providing me guidance and advice along the way. This study would not be possible without the training and support of the staff of both the Clemson Electron Microscope Facility and Clemson Light Imaging Facility, especially George Wetzel and Justin Scott. I would also like to acknowledge Dr. Matthew Blais of the Southwest Research Institute for his contributions in funding this research and guidance throughout the project. 


\section{TABLE OF CONTENTS}

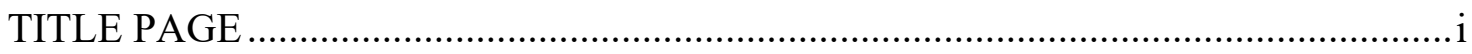

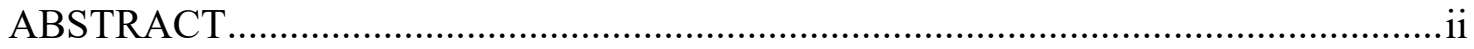

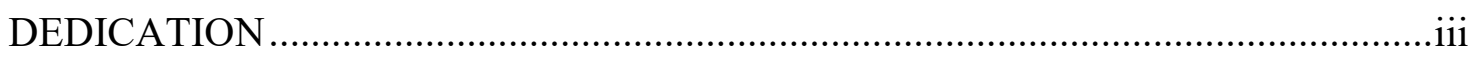

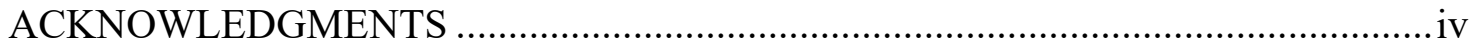

LIST OF TABLES .........................................................................................

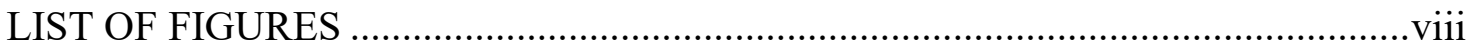

\section{CHAPTER}

I. INTRODUCTION AND STATE OF THE ART .................................. 1

Brief overview of chemical warfare agents ....................................... 1

State of the art in chemical weapon decomposition............................. 4

II. METHODS, RESULTS, AND DISCUSSION ...................................... 11

Materials and Methods

Selected compounds............................................................... 11

Safety Considerations .................................................................. 12

Pyrolyzed cotton balls.................................................................... 13

Set-up used for combustion experiments ...................................... 13

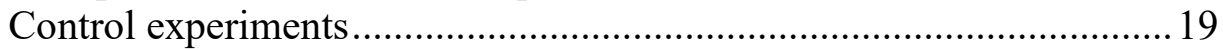

Pyrolyzed cotton ball characterization............................................2 21

Results and Discussion

Pyrolyzed cotton ball characterization...........................................22

Control experiments ..................................................................... 30

Decomposition of 2-chloroethyl ethyl sulfide (CEES) ........................35

Decomposition of triethyl phosphate (TEP) ................................... 38

III. CONCLUSIONS AND FUTURE RESEARCH ................................. 41

Conclusion of pyrolyzed cotton ball experiments ............................ 41

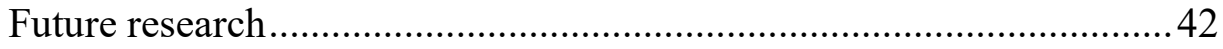


Table of Contents (Continued)

Page

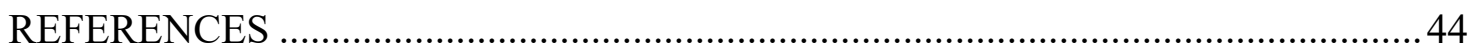




\section{LIST OF TABLES}

Table

Page

1.1 Table 1.1: Comparison of lethal doses $\left(\mathrm{LD}_{50}\right)$ for major

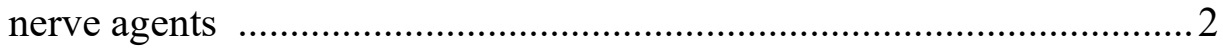

2.1 Maximum rates of burning for CEES in a $5.5 \mathrm{~cm}$ sample pan with various incendiary sources ............................................... 34

2.2 Maximum rates of burning for TEP in a $5.5 \mathrm{~cm}$ sample pan with various incendiary sources... 


\section{LIST OF FIGURES}

Figure

Page

2.1 Chemical warfare agents and associated simulants used in this study as proxies, chemical structures shown to highlight the similarities and differences with the agents and their associated simulants

2.2 Experimental set-up for the burning of chemical warfare agent simulants while measuring overflow. Fresh agent is pumped into the injection pan on the right while the napalm-coated pyrolyzed cotton balls were introduced to the sample pan on the left

2.3 Representative example of the experiment designed to measure the overflow of the simulant before and after the introduction of the napalm-coated PyCB to a $9 \mathrm{~cm}$ diameter sample pan containing CEES. The figure shows the initial overflow rate matching the input rate before (black) and after (orange) the ignition. The figure also shows the extrapolation of the initial overflow (dashed line) throughout the duration of the experiment.

2.4 Representative example of the difference between the input and output (combusted amount) of CEES simulant as a function of time utilizing pyrolyzed cotton balls as a substrate in a $9 \mathrm{~cm}$ diameter sample pan. This shows when the ignited napalmcoated pyrolyzed cotton ball is first introduced to the sample pan there is very little burning. After approximately 90 seconds, however, the rate of burning increases rapidly and approaches the maximum burn rate. During this period, the entire surface area of the sample pan is ignited, showing that the agent itself is serving as a fuel source for the combustion reaction 
List of Figures (continued)

Figure

Page

2.5 Scanning electron microscope images of unpyrolyzed (left) and pyrolyzed (right) cotton balls showing the increased number of fibers per unit area and the decreased size of the individual fibers as a result of the pyrolysis process

2.6 Scanning electron microscope images of unpyrolyzed (left) and pyrolyzed (right) cotton balls displaying the change in the structure of individual cellulose fibers as a result of the pyrolysis process

2.7 BET isotherm for pyrolyzed cotton balls indicating that there is a linear adsorption and desorption of nitrogen gas yielding a total surface area of $569.624 \mathrm{~m}^{2} / \mathrm{g}$

2.8 Pore size distribution in pyrolyzed cotton balls by the BJH method showing the majority of pores are centered around $19 \AA$....... 25

2.9 Image of pyrolyzed cotton ball with a drop of water supported on the surface due to the hydrophobic interaction between the pyrolyzed cotton ball fibers and the water droplet

2.10 Image of the results of the hydrophilic interaction between unpyrolyzed cotton ball fibers and water as the cotton ball was slowly retracted from the surface of the water, but continued wicking the water into the bulk of the cotton ball mass

2.11 Optical non-contact roughness measurements of interior structure of pyrolyzed cotton ball: (left) threedimensional rendering of the imaged area of the pyrolyzed cotton ball displaying the varied height of the fiber in reference to the objective; (right) superimposed height image on the two dimensional image of pyrolyzed cotton ball 
List of Figures (continued)

Figure

Page

2.12 Comparison of the maximum burning rates achieved with PyCBs in CEES as a function of the diameter of the sample pan. The size of the pan does initially influence the maximum rate of burning achieved, but as the pans continue to increase in size, the rate of burning plateaus.

2.13 Comparison of maximum burning rates achieved with PyCBs in TEP as a function of the diameter of the sample pan. The size of the pan appears to have minimal impact on the rate of burning achieved when TEP is used.

2.14 Proposed reaction mechanism for TEP generating flammable ethylene and the less toxic by-product, phosphoric acid. This reaction requires the high temperatures from the burning of napalm in order to liberate the ethylene and to proliferate the reaction until all ethyl groups are removed, leaving only phosphoric acid 


\section{CHAPTER ONE}

\section{INTRODUCTION AND STATE OF THE ART}

Chemical warfare agents are some of the most hazardous materials known to and created by man. These agents cause a wide variety of damage on the human body, ranging from superficial chemical burns to paralysis of the nervous system and death. Many different chemical agents have been developed over the years, and militaries have countered these developments with various means of decontamination and individual protection. Individual decontamination and protection methods have greatly improved over the past 100 years of conventional conflict and use of chemical warfare agents. These developments range from the rudimentary mask and filters developed during World War $\mathrm{I}^{1}$ to the currently researched protective suits and uniforms embedded with highly sophisticated metal organic framework particles capable of degrading potential contaminants. ${ }^{2,3}$ On the other hand, advancements in decontamination methods for decomposing bulk quantities of chemical agents has remained relatively stagnant. Seeking to address this gap in development, this manuscript presents a novel method which utilized pyrolyzed cotton balls (PyCBs) as a wick supporting the thermal degradation of multiple classes of chemical warfare agents.

\section{Brief overview of chemical warfare agents}

Chemical warfare agents (CWAs) were employed in modern conflicts dating back to World War I when sulfur mustard (bis(2-chloroethyl) sulfide), also known as HD, was

first used to incapacitate large numbers of soldiers. ${ }^{1,4,5}$ Sulfur mustard's primary method 
of incapacitation is through the production of highly unstable compounds, such as sulfonium, which then alkylate sulfhydryl or amino groups in proteins and nucleic acids. $^{4,6}$ This alkylation proceeds to cause further damage by preventing cellular glycolysis and eventually leading to necrosis, typically within an hour of exposure to the agent. ${ }^{4}$ Despite its relatively low fatality rate,,${ }^{4,7,8}$ exposure to even small doses of HD can cause extensive damage to the skin, eyes, and respiratory systems (i.e. blisters, swelling, and necrosis). ${ }^{4,6}$

In contrast to HD, nerve agents such as $o$-ethyl N,N-dimethyl phosphoramido cyanidate (Tabun or GA), (R,S)-propan-2-yl methylphosphonofluoridate (Sarin or GB), and ethyl ( \{2-[bis \{propan-2-yl)amino]ethyl $\}$ sulfanyl)(methyl)phosphinate (VX) are considered to be extremely lethal and have the capability of killing large numbers of people within minutes at the extremely low doses of $14 \mathrm{mg} / \mathrm{kg}$ (of body weight), 24 $\mathrm{mg} / \mathrm{kg}$, and $0.04 \mathrm{mg} / \mathrm{kg}$ respectively. ${ }^{9-11}$ These nerve agents primarily interact with the muscular and nervous systems of the body by binding to the enzyme, acetylcholinesterase $(\mathrm{AChE})$, and preventing the reuptake of the neural transmitter, acetylcholine, in the synaptic junction. ${ }^{10}$ The accumulation of acetylcholine causes muscular and respiratory failure within minutes if the agent is inhaled and within hours if the agent is absorbed through the skin. ${ }^{12,13}$ The lethal dose $\left(\mathrm{LD}_{50}\right)$ for some common nerve agents is shown below in Table 1 for both air concentration and dermal exposure.

Table 1.1: Comparison of lethal doses ( $\left.\mathrm{LD}_{50}\right)$ for major nerve agents ${ }^{10,14}$

\begin{tabular}{cccc} 
& Tabun (GA) & Sarin $(\mathrm{GB})$ & VX \\
\hline $\begin{array}{c}\text { Dermal liquid } \\
(\mathrm{mg} / \mathrm{kg})\end{array}$ & $14-21$ & 24 & 0.04 \\
$\begin{array}{c}\text { Inhalation } \\
\left(\mathrm{mg}-\mathrm{min} / \mathrm{m}^{3}\right)\end{array}$ & $150-400$ & $70-100$ & $30-36$
\end{tabular}


In addition to requiring only miniscule concentrations to cause catastrophic effects, some nerve agents are considered environmentally persistent due to their low volatility and poor solubility in water. ${ }^{10,15,16}$ Furthermore, the agents are resistant to hydrolysis within the body and therefore remain in the system for long periods of time, even at doses below the lethal dose. ${ }^{10} \mathrm{VX}$ is especially toxic, not only because of the significantly lower concentrations which are lethal compared to the other nerve agents, but also because it does not experience any significant breakdown in the body once it binds to acetylcholinesterase. ${ }^{17}$ This means that even extremely small doses of VX can have long-term, irreparable health impacts.

In 1997, the Chemical Weapons Convention (CWC) established the Organization for the Prohibition of Chemical Weapons (OPCW) which prohibited the use and production of CWAs. Unfortunately, these efforts have not prevented non-state actors such as terrorist organizations and rogue nation-states from developing and/or utilizing these incredibly destructive weapons. Two of the most recent, highly publicized attacks occurred using V class nerve agents: 2017 in an assassination in Kuala Lumpur and 2018 during a failed assassination attempt in the United Kingdom. On a greater scale, both chlorine-based and sarin agents were used against both military and civilian populations with devastating effects during the Syrian civil war, which started in $2012 .{ }^{18}$ Based on the high degree of damage these agents can cause at extremely low concentrations, it is necessary to develop faster, inexpensive, and more effective means to decompose CWAs in large volumes. 


\section{State of the Art in Chemical Weapon Decomposition}

The environmental conditions (i.e. temperature, humidity, and wind) have a significant effect on the persistence of CWAs in an environment. Both HD and GB are considered moderately persistent due to extended degradation times under ambient conditions (i.e. $\sim 30$ hours for $\mathrm{HD}^{19}$ and $\sim 65$ hours for $\mathrm{GB}^{10,20}$ ). These factors contribute to chemical treatment methods being the primary means of decomposing both of these classes of CWAs. While there has been recent research into both physical ${ }^{21}$ and combinations of chemical and physical methods ${ }^{22,23}$, the majority of decontamination efforts remain focused on chemical decontamination.

In 1992, the United States Army sought to develop multiple methods for disposing of its chemical weapon stockpile with a focus on incineration or chemical neutralization. ${ }^{24}$ Neutralization methods were initially abandoned, however, due to incomplete reactions and the large volumes of chemical waste produced. Instances were reported by the US Army's Chemical Weapon Disposal Program (CWDP) where reactions that were expected to take only hours required days to complete. ${ }^{24,25}$ These extended time scales were attributed to the sensitivity of neutralization methods towards both $\mathrm{pH}$ and temperature as well as equilibrium processes that occurred. ${ }^{24}$ Subsequently incineration was selected as the preferred method for large scale disposal. ${ }^{25}$ Incineration and thermal decomposition methods remained the primary route for the U.S. Army to destroy large volumes of agent throughout the 1980s and 1990s. ${ }^{24,25}$ The Army's plans to decompose the United States' chemical weapons stockpile through incineration were abandoned in the 1990s however, partially due to the large cost associated with building 
facilities that could incinerate the agents at each of the chemical weapon storage facilities across the country. ${ }^{24,25}$ While incineration at high temperatures is effective at treating large stockpiles, it is both a time and cost intensive process due to the equipment and materials that are required to achieve incineration of both the agent and the storage materials. The U.S. Army estimated that it would take more than 5 years at some of the chemical storage facilities to complete the decontamination process through large scale incineration. ${ }^{24}$ These restraints also limit the effectiveness of current incineration methods towards field expedient or on-site destruction of chemical weapons that may be discovered during either armed conflict or by regulatory agencies during inspections.

Metal organic frameworks combine a metal central node, commonly referred to as the secondary building unit, with organic linker molecules to create particles with a repeating structure similar to a crystal lattice. These structures have been compared to enzymes due to their ability to be reactive towards a specific target molecule or functional group. Unlike enzymes however, MOFs are not restricted to a single active site on each molecule, but have numerous active sites, both on the surface and throughout the internal structure of the particle. ${ }^{26}$ Owing to these factors, recent research has found that metal organic frameworks (MOFs) can effectively degrade a variety of chemical warfare agents when applied to a substrate before exposure to CWAs. ${ }^{23,27,28}$ Metal organic frameworks are uniquely positioned for the decomposition of chemical warfare agents because they combined the high surface area desirable of heterogeneous catalysts with the highly functionalized nature of organic molecules. ${ }^{26,29,30}$ While MOFs have a wide variety of applications, the most commonly studied structures for the decomposition 
of chemical warfare agents are those containing a zirconium metal node, such as UiO-66, UiO-67, NU-1000, and MOF-808. ${ }^{31-34}$ All of these MOFs have been reported to rapidly catalyze the decomposition of dimethyl 4-nitrophenol phosphate (DMNP), a common simulant for sarin and VX, with half-lives on the order of minutes. ${ }^{31,33}$ Despite the high efficiency of these molecules, however, there have been no reported studies of MOFs being applied to a bulk decontamination process. Instead the focus of these methods appears to center around the utility of MOFs to be embedded on the surface of different textiles for use in individual protective suits and clothing. ${ }^{2,3,35}$ Additionally, due to the high surface area of the MOFs and their ability to be used as heterogeneous catalysts there is potential for them to be incorporated into filters, such as in water treatment or air filtration systems. ${ }^{2}$ Similarly, another physical decontamination method which has recently received interest is in the use of highly oxidized porous carbon surfaces to decompose simulants for HD up to $80 \%$ decomposition after 24 hours of contact. ${ }^{36}$ While both MOFs and oxidized carbon yield significant potential in the realm of physical decontamination and personal protection, there appears to be limited application for these methods in the treatment of bulk stores of agents.

Currently, the predominant methods to degrade large scale quantities of CWAs are through chemical treatment. ${ }^{37}$ Of these methods, hydrolysis and oxidation are among the most common methods currently used to decompose chemical agents..$^{1,22,37-40}$ While these approaches are generally effective, their efficiency is heavily dependent on the reaction conditions (i.e. $\mathrm{pH}$ and temperature), and they also require a large excess of reagents to achieve acceptable levels of decontamination. ${ }^{20,38}$ In addition, these processes 
also generate large volumes of waste and a number of toxic by-products. ${ }^{19,20,38,41}$ Aiming to improve the rate and/or yield of these reactions, recent studies of the hydrolysis of CWAs have focused on the use of catalysts, such as silver nanoparticles or alumina substrates. ${ }^{41,42}$ While these studies are promising, the development of nanoparticles is costly, both in terms of money and time.

Due to the poor performance of neutralization techniques employed by the United States Army in the 1980s, supercritical water oxidation (SCWO) was examined as an alternate technology for the potential large-scale decontamination. ${ }^{24}$ The U.S. Army conducted research into supercritical water oxidation technology during the late 1980s and early 1990s, and concluded that the process was feasible for the decomposition of chemical warfare agents. ${ }^{24}$ At the time, there were two major concerns which prevented the U.S. Army from pursuing this method for the stockpile disposal program. First, the technology was still in its nascent stage with only prototypes developed. These prototypes were shown to be effective at degrading various organic hazardous wastes, however they had not been used on any chemical warfare agents. Secondly, the method would only apply to the actual chemical warfare agents, but could not be applied to the storage containers and munitions which also needed to be decontaminated. The U.S. Army did acknowledge that this technology would have significant advantages over incineration of the chemical agents if the technology were fully developed and applied to chemical weapons. ${ }^{24}$

While there were advances in the supercritical water reactor technology throughout the 1990s and early 2000s, there was very little research into the application 
towards chemical weapons. In 2007 a group at the Korea Institute of Science and Technology led by Jae-Duck Kim developed a bench scale super critical water reactor which they claimed was able to decompose chemical warfare agent precursors with high efficiency. ${ }^{43}$ For this study they chose to use precursors that are used in the binary preparation of chemical agents, instead of the actual chemical agents or other frequently utilized simulants for the agents. The precursors examined in this study were $\mathrm{OPA}^{44}$, a mixture of iso-propyl amine and iso-propyl alcohol, and thiodiglycol (TDG). OPA is part of the binary precursor which is mixed with methyl phosphonic difluoride (DF) to produce the chemical agent sarin $(\mathrm{GB}){ }^{44}$ TDG is part of the binary precursor that is combined with di-iso-propyl aminoethylmethyl phosphite $(\mathrm{QL})$ to produce the chemical agent VX. TDG was specifically chosen as one of the model simulants for the study as corrosion of the reactor due to the production of sulfuric acid and hydrogen sulfide during oxidation would prevent upscaling of the technology and its application towards to degradation of any chemical warfare agents containing sulfur-based functional groups, such as HD and VX. ${ }^{43}$ Through these experiments it was shown there was no evidence of corrosion on the metallic components of the reactor from any sulfuric acid or hydrogen sulfide species generated. From these results it was concluded that a SCWO reactor for the treatment of chemical warfare agents such as $\mathrm{HD}$ or VX required a reactor vessel fabricated with components made from titanium, nickel, and molybdenum. ${ }^{43}$ While this system did display extremely high efficiency with conversions of over $99 \%$ of both OPA and TDG, there were no reported studies performed on the true chemical warfare agents or the other components of the binary precursors. 
Similarly, in 2002 a study investigated if VX could be decomposed by SCWO. While this study did use the actual chemical agent, the VX was pretreated with $\mathrm{NaOH}$ prior to being injected into the reactor. While the SCWO was effective at degrading $97 \%$ of the VX byproducts, this approach is a secondary step for the removal of the hazardous byproducts that are produced by the neutralization reaction with $\mathrm{NaOH} .{ }^{45}$ This example shows that there is considerable value in the technology and its implementation in CWA disposal programs, however based on the current research, super critical water oxidation needs further study in order to determine if similar conversion percentages can be achieved.

Despite the advances in hydrolytic decomposition and its potential to decontaminate equipment with high efficiency, ${ }^{41}$ a method for the thermal degradation of bulk volumes of agents, such as in stockpiles, with limited sample preparation or treatment has not been widely studied. ${ }^{46,47}$ Possible reasons for the dearth of research on this strategy include the low-flammability of some of these compounds as well as the risk of producing hazardous by-products or even converting the agent into the gaseous phase if the reaction conditions are not well-controlled. ${ }^{48}$ In addition, the specific instrumentation and low-scale volumes (less than $20 \mu \mathrm{L}$ in previous studies ${ }^{46,47}$ ) have not provided enough information to support field-deployment of this strategy with the aim of decomposing large volumes of chemical agents.

Aiming to address these limitations, this study presents a simple approach to facilitate the decomposition of a variety of CWAs in large volumes via open-flame combustion. The approach is based on the use of pyrolyzed cotton balls acting as wicks, 
which upon being soaked with napalm B as the starting incendiary agent, are able to drive the decomposition to be self-sustaining (i.e. using the agent itself to fuel the combustion) under ambient conditions. The method represents a low-cost alternative to achieve combustion and thermal degradation of liquid chemical warfare agents which can be scaled to accommodate bulk volumes of agent. While the proposed method does not remove the requirement to process and clean the resulting smoke and atmosphere of potentially harmful byproducts, it does eliminate the need for a large-scale industrial furnace. 


\section{CHAPTER TWO}

\section{METHODS, RESULTS, AND DISCUSSION}

\section{Materials and Methods}

\section{Selected compounds}

Due to the toxicity of chemical warfare agents and the extensive requirements necessary for their use in laboratory research, this study focused on the use of simulants in place of the agents themselves. These compounds have chemical structures and reactivity similar to the chemical warfare agents they model, but with much lower toxicity. Figure 2.1 depicts common chemical warfare agents and their associated simulants. ${ }^{37,49}$ It is also important to note that the use of simulants is common practice when studying chemical warfare agents, and multiple studies have shown reactions with live agents generally proceed at a much higher rate than reactions with the simulants due to the presence of more reactive functional groups. ${ }^{20,37,50-52}$ Therefore, we focused on the simulant 2-chloroethyl ethyl sulfide (CEES) for the blister agent sulfur mustard (HD) and triethyl phosphate (TEP) for the nerve agent sarin (GB). The structures and full IUPAC naming conventions for the chemical agent and the associated simulants examined in this study are shown below in Figure 2.1. The simulants used in this study were purchased from either Sigma-Aldrich (St. Louis, MO) or TCI America (Portland, OR). All products were in $97.0 \%$ purity or greater and used as received. Napalm B, the incendiary agent in this study, was prepared by first mixing approximately $70 \% \mathrm{v} / \mathrm{v}$ gasoline (93 octane) and 30\% v/v benzene (Sigma Aldrich; St. Louis, MO). Then, polystyrene (from common 
packing peanuts and Styrofoam) was added to the solution as a thickening agent at a ratio of $0.195 \pm 0.002 \mathrm{~g}$ polystyrene (three packing peanuts) to $1 \mathrm{~mL}$ of the gasoline/benzene mixture. Since the solvent is volatile, a fresh sample was prepared daily. It is important to note that other forms of polystyrene will work for the preparation of napalm B, however our group found that packing peanuts dissolve faster in the solution than polystyrene pellets with no decrease in flammability.

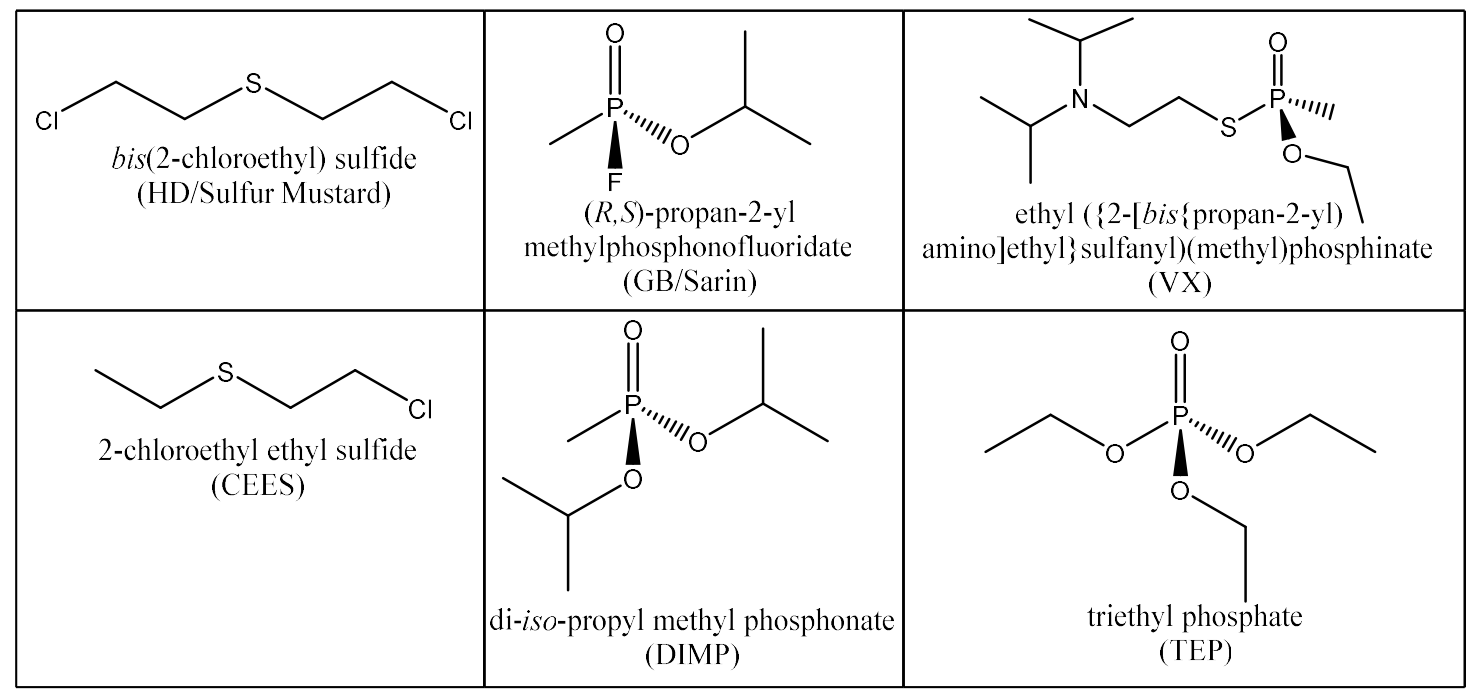

Figure 2.1: Chemical warfare agents and associated simulants used in this study as proxies, chemical structures shown to highlight the similarities and differences with the agents and their associated simulants

\section{Safety considerations}

Although the described experiments are performed with simulants (not live chemical warfare agents), these compounds are still toxic and can cause blisters and severe injuries. Extreme caution must be exercised when handling these compounds. In order to avoid possible injuries, all experiments were performed in a vented hood, where all agents and waste products were properly contained. 
Pyrolyzed Cotton balls

Cotton balls (Up \& Up brand, purchased from a local convenience store) were selected to serve as the substrate for the delivery of the incendiary agent due to their low weight, high cellulose content, and 3-dimensional structure. In order to increase the hydrophobicity while maintaining the structure of the material, the cotton balls were pyrolyzed using a tube furnace (Thermolyne F21135, Barnstead International, Dubuque, IA) following a previously reported procedure. ${ }^{53}$ The cotton balls were placed in the center of the furnace over a silicon wafer which acts as an inert holder. The ends of the quartz tube were then sealed and flushed for 5 minutes with forming gas $\left(1 \mathrm{~L}\right.$. $\min ^{-1} 95 \%$ Ar, $5 \% \mathrm{H}_{2}, \mathrm{v} / \mathrm{v}$ ) to remove the ambient air contained in the tube and generate a mildly reducing environment. ${ }^{53} \mathrm{Next}$, the temperature was increased to $1000{ }^{\circ} \mathrm{C}$ at $30{ }^{\circ} \mathrm{C} \cdot \mathrm{min}^{-1}$. After 1 hour at $1000{ }^{\circ} \mathrm{C}$, the system was allowed to cool to room temperature and the resulting pyrolyzed cotton balls (PyCBs) were stored in petri dishes until used.

\section{Set-up used for combustion experiments}

In order to perform the combustion experiments and follow the reaction in real time, the experimental set-up shown in Figure 2.2 was developed. The system comprised of a piston pump (NE-3000 Just Infusion Syringe Pump, New Era Pump Systems Inc, Farmingdale, NY) used to deliver the simulants, a $50 \mathrm{~mL}$ glass syringe (Fortuna Optima 7.140 Luer-Lock tip, Poulten \& Graf GmbH, Wertheim, Germany), two stainless steel pans connected by plastic tubing, a calcium carbonate fire barrier, an overflow tube, a 
collection pan, and a digital balance (Mettler Toledo ME104E, Zaventem, Belgium)

connected to a computer via a standard serial cable using the accompanying balance software (LabX Direct Balance, version 2.5). Thermal images and videos were captured using an infrared camera (FLiR One Pro iOS version, FLIR Systems, Inc., Wilsonville, OR).

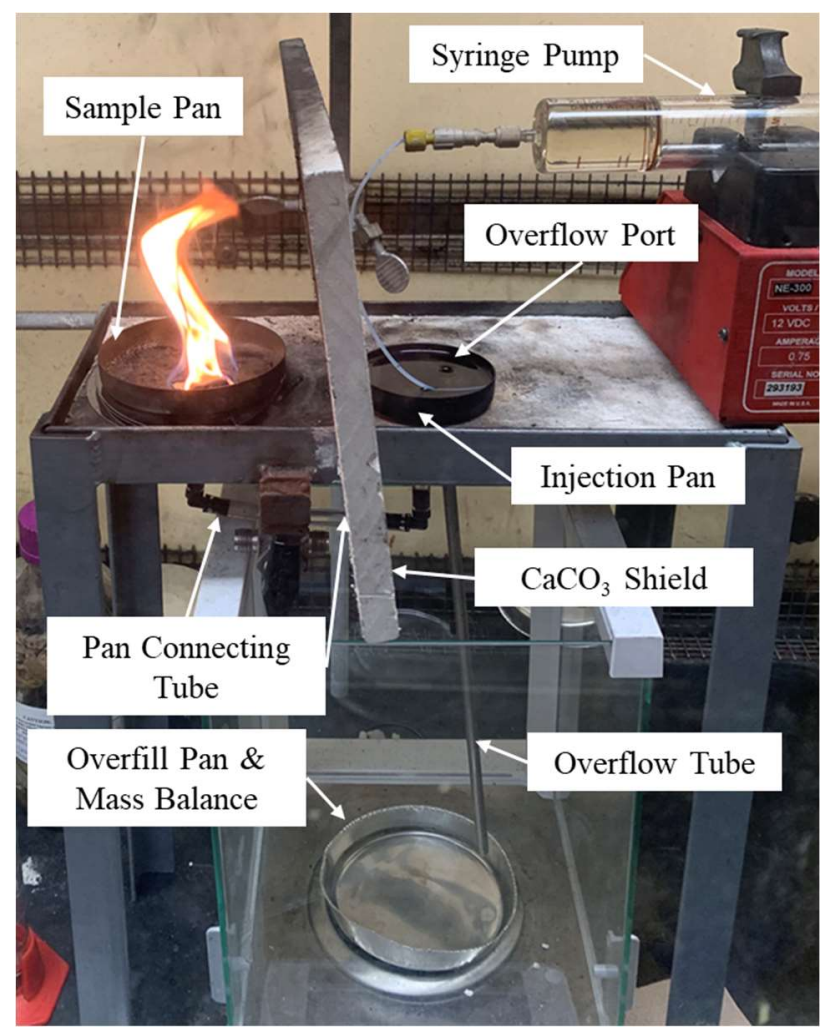

Figure 2.2: Experimental set-up for the burning of chemical warfare agent simulants while measuring overflow. Fresh agent is pumped into the injection pan on the right while the napalm coated pyrolyzed cotton balls were introduced to the sample pan on the left.

Before each experiment, all pans and tubes were cleaned with methanol and allowed to dry completely in order to prevent contamination. The syringe was then loaded with $30-40 \mathrm{~mL}$ of the selected agent (i.e. simulant) to be studied. The syringe was 
secured to the pump which was programmed for a delivery rate of $4.00 \mathrm{~mL} \cdot \mathrm{min}^{-1}$. Next, $\sim 10 \mathrm{~mL}$ of the selected simulant was dispensed into the injection pan (right pan in Figure 2.2) and allowed to equilibrate with the sample pan (left pan in Figure 2.2) via the connection tubing underneath the pans. Any excess agent was drained via the overflow tube, into the collection pan, which was placed on the balance. This pre-filling step reduced the overall time of the experiment by eliminating the necessity of reloading the syringe after the simulant equilibrated and filled up both pans to the level of the overflow tube. It is also important to note that the total volume of sample used depended on the diameter of the pans. To limit the variance in the data a constant pumping rate was used in all experiments and each iteration had an overflow period as an internal standard to compare the rate of overflow before and after burning was achieved. For the experiments described herein the only variables were the diameter of the sample pan and the type of simulant used.

In all experiments, the change in the mass from the overflow of simulant (i.e. from the injection pan and sample pans overflowing into the collection pan) was recorded as a function of time. This approach allowed for the determination of both the initial delivery rate prior to the initiation of combustion whereby all of the sample overflowed into the collection pan, and the burning rate where the difference between the accumulated mass in the collection pan and the amount delivered into the system. A representative example of the data acquired is shown in Figure 2.3 and Figure 2.4 (vide infra), where the mass in the overflow pan is recorded as a function of time. 


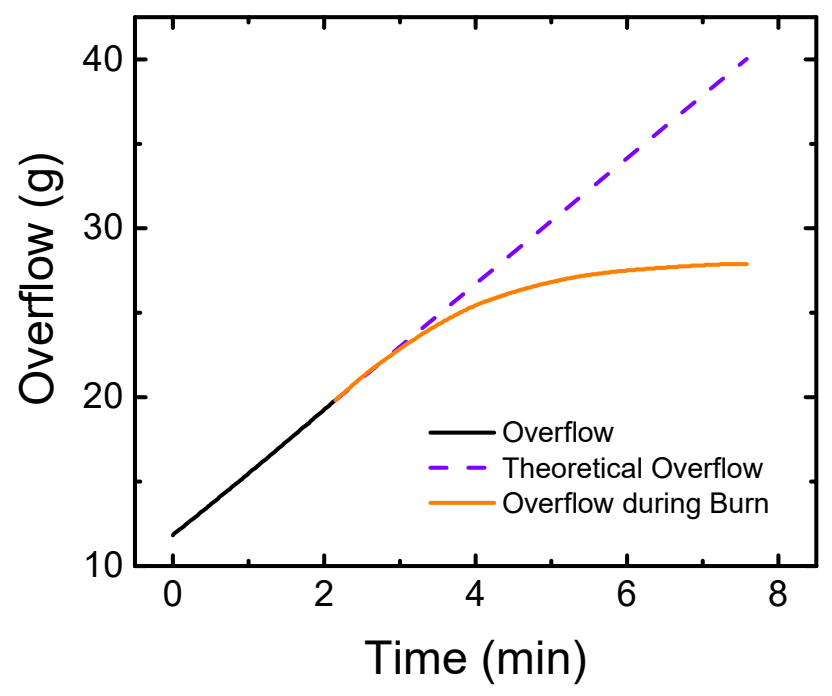

Figure 2.3: Representative example of the experiment designed to measure the overflow of the simulant before and after the introduction of the napalm coated PyCB to a $9 \mathrm{~cm}$ diameter sample pan containing CEES. The figure shows the initial overflow rate matching the input rate before (black) and after (orange) the ignition. The figure also shows the extrapolation of the initial overflow (dashed line) throughout the duration of the experiment.

As it can be observed in Figure 2.3, a linear increase in the overflow mass was observed at the beginning of the experiment, when the agent is pumped at a constant rate prior to ignition. This initial rate (solid black line, Figure 2.3) corresponds to the pumping rate of $4.00 \mathrm{~mL}$. $\mathrm{min}^{-1}$. Across all of the pans examined in this study, the average rate of overflow was $3.6 \pm 0.5 \mathrm{~mL}$. $\mathrm{min}^{-1}$, with the smaller pans at $4.00 \mathrm{~mL}$. $\mathrm{min}^{-1}$ rate and the larger pans at $3.25 \mathrm{~mL} . \mathrm{min}^{-1}$ rate. This difference was expected due to the increased volumes in the larger sample pans necessitating the pumping of more solution into the system in order to sustain combustion.

Once the ignited napalm-coated PyCB was introduced to the sample pan (after approximately 2 min as shown in Figure 2.3), the simulant started to burn, and a clear decrease in the overflow mass was observed, as the simulant pumped into the system was 
burnt and the rate of overflow decreased. By comparing the overflow rate with the pumping rate - extrapolated for the duration of the experiment (dashed line, Figure 2.3) the amount and rate of the burning for the agent can be determined. The maximum burning rate in each experiment was compared to the overflow rate before the ignited pyrolyzed cotton ball was introduced to the sample pan, in order to minimize the effects of variables such as small variations in the initial temperature of the pans and/or connecting lines. This internal comparison allowed for a more accurate determination of the rate of burning for each experiment versus using the average rate of overflow for each pan size. These results were used to calculate the difference between the input (i.e. overflow without burning) and the output (i.e. overflow with burning) amounts, which represents the mass of the simulant burnt during the entire burning period, as shown in Figure 2.4. 


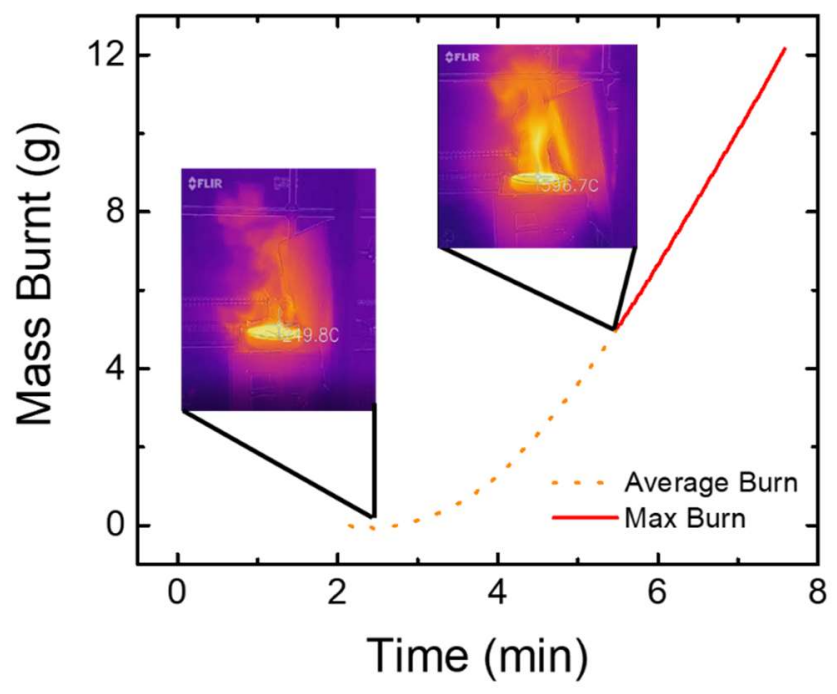

Figure 2.4: Representative example of the difference between the input and output (combusted amount) of CEES simulant as a function of time utilizing pyrolyzed cotton balls as a substrate in a $9 \mathrm{~cm}$ diameter sample pan. This shows when the ignited napalm-coated pyrolyzed cotton ball is first introduced to the sample pan there is very little burning. After approximately 90 seconds, however, the rate of burning increases rapidly and approaches the maximum burn rate. During this period, the entire surface area of the sample pan is ignited, showing that the agent itself is serving as a fuel source for the combustion reaction.

It is important to note that when the napalm-coated pyrolyzed cotton ball was ignited and introduced into the simulant, the change in overflow rate was attributed to the combustion of napalm. In control experiments where pyrolyzed cotton balls and napalm were burned without the presence of any chemical warfare agent or simulant the pyrolyzed cotton balls and napalm burned for approximately 4 minutes. The selfextinguishing of the flame at this stage was attributed to the consumption of the napalm incendiary agent. It was observed that during these experiments the flame was restricted to only the pyrolyzed cotton ball and did not spread across the surface area of the pan. As the energy and heat from this combustion of napalm was transferred the simulant was able to reach its respective ignition temperature and a sustained combustion was 
achieved. The period of time before the sustained burning occurred was referred to as the initial burn period, as the burning was not completely fueled by the agent (orange dotted line, Figure 2.4). The period of time where a constant burning rate - as indicated by the linear region of the burnt mass curve (solid red line, Figure 2.4) - was referred to as the maximum burning rate. The resulting data were evaluated for each experiment in order to determine the rate of maximum burning for the different simulants as a function of the sample pan diameter. In all cases, data collection continued until either no simulant remained in the syringe pump, or the combustion in the burn pan self-extinguished. One shortfall of this experimental set-up is that it allows for determining the rate of burning for a variety of CWA simulants, but not for determining the percentage of CWA decomposed. This is due to the dynamic nature of the experimental set-up. Further studies and new experimental designs are needed to determine and optimize the overall decomposition percentage before the methods could be applied to scale. These studies were outside of the scope of this research for achieving combustion and measuring rate as a function of surface area.

\section{Control experiments}

In order to show that the increase in the rate of burning was a result of the pyrolyzed cotton ball wick, several control experiments were conducted. The first experiment used napalm added to the pool of simulant in a dropwise manner. In this experiment a portion of the napalm mixture was scooped onto the end of a spatula, 
suspended over the pool of simulant, and ignited. Once the napalm sample was ignited it dripped into sample pan and the experiments continued as described above in Figure 2.2.

Controls were also conducted with napalm spread on a wire mesh and suspended above the surface of the agent in the sample pan. This experiment allowed for the determination of the impact that the incendiary agent alone had on inducing burning of the sample. In order to ensure accuracy, pyrolyzed cotton balls were coated with napalm and then weighed to determine the average amount of napalm that a pyrolyzed cotton ball could support. It was found that one pyrolyzed cotton ball supported $1.2 \pm 0.1 \mathrm{~g}$ of napalm. The wire mesh was then coated with $1.2 \pm 0.1 \mathrm{~g}$ of napalm, placed on the sample pan, and ignited once the syringe pump was turned on and a consistent rate of overflow was established. This control was applied for both simulants examined in this study, CEES and TEP.

In order to compare the impact of pyrolyzing cotton balls it was necessary to establish the rate of burning with unpyrolyzed cotton balls. In the first control with unpyrolyzed cotton balls, untreated cotton balls were ignited and placed in the sample pan as previously described. All other conditions were the same as previously described. In the second control, unpyrolyzed cotton balls were coated with napalm, ignited, and then placed in the sample pan as previously described. While the unpyrolyzed cotton balls were slightly burned due to the combustion of the napalm the absence of a reducing atmosphere prevents the unpyrolyzed cotton balls from undergoing pyrolysis. All other conditions were the same as previously described. These controls were applied for both simulants examined in this study, CEES and TEP. 
Finally, in order to examine the impact of the structure of the pyrolyzed cotton balls on inducing burning, a pyrolyzed cotton ball was ground into a fine powder with a mortar and pestle. This powder was then added to $1.2 \pm 0.1 \mathrm{~g}$ of napalm and thoroughly mixed. The suspension was then dried for 30 minutes and formed into a spherical pellet with a diameter of approximately $1 \mathrm{~cm}$. Pellets were then ignited and placed in the sample pan as previously described. All other conditions were the same as previously described. These controls were applied for both simulants examined in this study, CEES and TEP.

It is important to note that other sources of cellulose were examined in this study in order to determine if the starting material was critical to inducing burning. Whatman type 3 chromatography paper was pyrolyzed in the same method as previously described for the cotton balls. The resulting pyrolyzed paper was not able to support the napalm mixture and no measurable burning was detected in these experiments.

\section{Pyrolyzed cotton ball characterization}

Pyrolyzed cotton ball samples were characterized for their surface area, average pore diameter, and physical structure in order to determine the effect that pyrolyzing the cotton balls had on the ability of the substrate to wick the simulants. Surface area and pore diameter measurements were conducted via the widely accepted method of nitrogen adsorption isotherms and the application of the Brunauer-Emmett-Teller (BET) and Barrett-Joyner-Halenda (BJH) methods respectively. ${ }^{54,55}$ The nitrogen adsorption isotherms and pore size analyses was conducted using an Autosorb IQ (Quantachrome 
Instruments, Ashland, VA). Samples were outgassed under vacuum for 5 hours at $300{ }^{\circ} \mathrm{C}$ in order to remove any impurities from sample pores. Scanning electron microscope (S3400 and SU6600, Hitachi High Technologies, Pleasanton, CA) imaging was conducted in order to compare changes in the physical structure of the cotton balls as a result of the pyrolyzation process.

Optical imaging was conducting at the Clemson Light Imaging Facility (CLIF) by a trained technician with an Olympus OLS5000 LEXT optical profiler (Olympus Corporation, Tokyo, Japan). Through this analysis it was possible to determine the roughness of the pyrolyzed cotton balls and the width of the fibers. In this study a pyrolyzed cotton ball was segmented into six pieces in order to confirm the consistency of the structural features throughout the pyrolyzed cotton ball. While it was not possible to construct a three-dimensional rendering of the pyrolyzed cotton ball, through this imaging technique it was possible to view the layering and interconnections between the individual strands of the pyrolyzed cellulose fibers.

\section{Results and Discussion}

Pyrolyzed cotton ball characterization

In order to confirm the structural change of the cotton balls as a result of the pyrolysis process, both untreated cotton balls and pyrolyzed cotton balls were investigated via scanning electron microscopy (Figure 2.5). The unpyrolyzed cotton ball is composed of large fibers ( $16 \pm 4 \mu \mathrm{m}$ in diameter) entangled to support the 3dimensional structure. Upon pyrolysis the overall structure of the cotton ball was 
preserved, but a significant reduction in size $(54 \%$, from $3.2 \pm 0.2 \mathrm{~cm}$ to $1.7 \pm 0.2 \mathrm{~cm})$

and mass $(92 \%$, from $0.650 \pm 0.030 \mathrm{~g}$ to $0.052 \pm 0.003 \mathrm{~g})$ was observed.
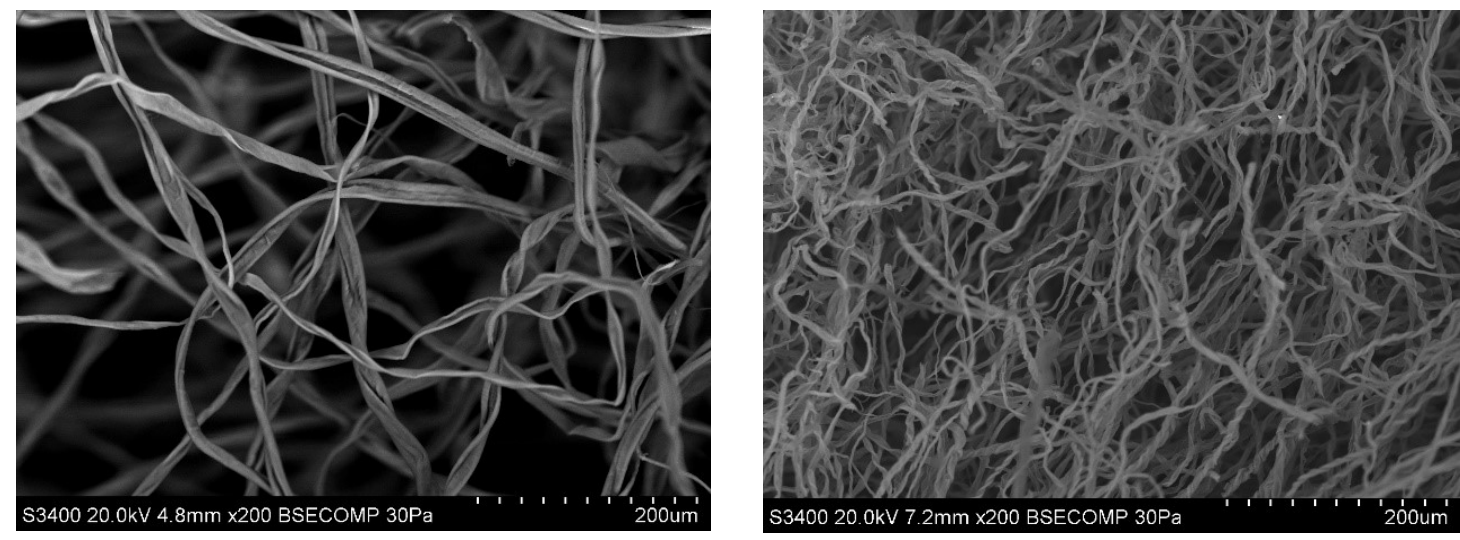

Figure 2.5: Scanning electron microscope images of unpyrolyzed (left) and pyrolyzed (right) cotton balls showing the increased number of fibers per unit area and the decreased size of the individual fibers as a result of the pyrolysis process.
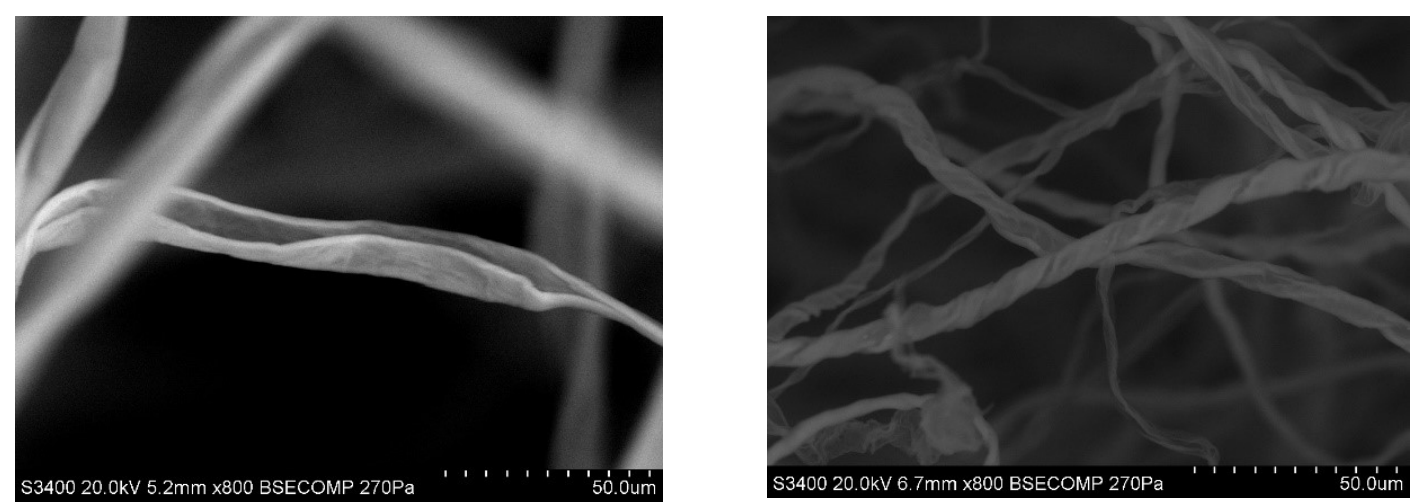

Figure 2.6: Scanning electron microscope images of unpyrolyzed (left) and pyrolyzed (right) cotton balls displaying the change in the structure of individual cellulose fibers as a result of the pyrolysis process

This decrease in the overall mass of the pyrolyzed cotton balls was attributed to the cleavage of low molecular weight oxygenated groups from the cellulose structures and the coalescence of the overall structure. ${ }^{53}$ The resulting PyCB is therefore composed of a more rigid yet brittle three dimensional network of carbon fibers with an average size of $14 \pm 1 \mu \mathrm{m}$. It is important to note that this small difference is not indicative of the 
dimensional changes occurring in each fiber, as the fibers in the PyCB appear to be twisted in comparison with the generally flat shape of the fibers observed in the unpyrolyzed cotton ball. This difference limits our ability to accurately measure the true decrease in the size of the individual fibers due to pyrolysis. As shown in Figure 2.6 there is a significant change in the structure of the individual cellulose fibers as a result of the pyrolysis process. This is believed to both increase the surface-area-to-volume ratio of the individual fibers and contribute to the changes in the hydrophobicity of the resulting cellulose fibers. These structural changes contribute to the ability of the pyrolyzed cellulose fibers to support the combustion of the CWA simulants, while the unpyrolyzed cellulose was rapidly extinguished when exposed to liquid CWA simulants.

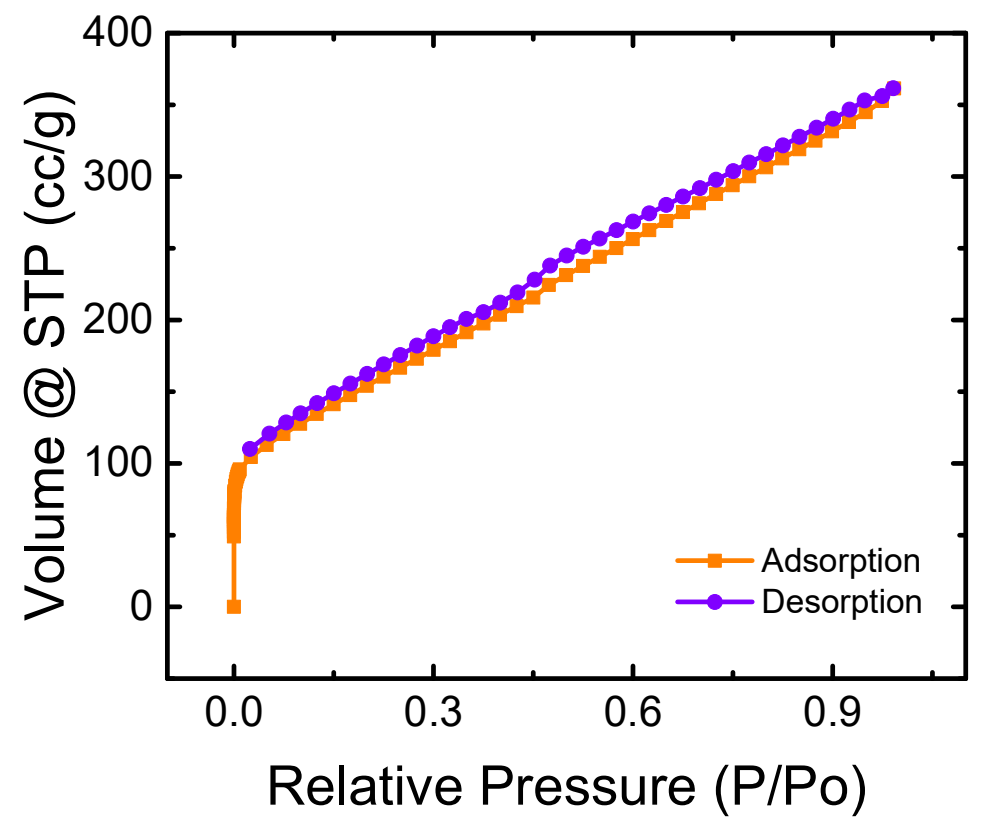

Figure 2.7: BET isotherm for pyrolyzed cotton balls indicating that there is a linear adsorption and desorption of nitrogen gas yielding a total surface area of $569.624 \mathrm{~m}^{2} / \mathrm{g}$ 
The surface area of the pyrolyzed cotton balls was calculated using the BrunauerEmmett-Teller (BET) method which revealed a surface area of $569.624 \mathrm{~m}^{2} / \mathrm{g}$ (Figure 2.7). This surface area suggests that the pyrolyzed cotton balls are between mesoporous carbon and activated carbon structures. ${ }^{54,55}$ Furthermore, the Barrett-Joyner-Hallenda $(\mathrm{BJH})$ method revealed the pore distribution of the pyrolyzed cotton balls centered around $19 \AA$ (Figure 2.8). This small pore size contributes to the pyrolyzed fibers to serve as a wick in lieu of an absorbent as the polar surface areas of both GB $\left(24.3 \AA^{2}\right)$ and the simulant TEP $\left(44.8 \AA^{2}\right)$ are larger than the pores.

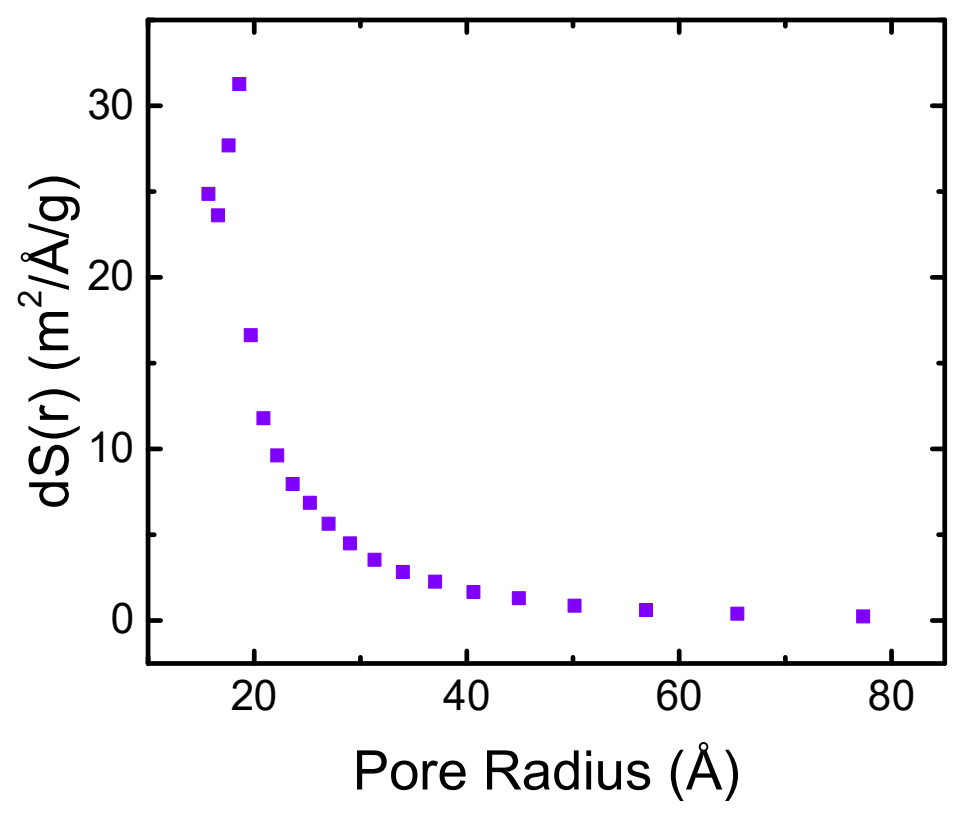

Figure 2.8: Pore size distribution in pyrolyzed cotton balls by the BJH method showing the majority of pores are centered around $19 \AA$

Finally, the pyrolysis process makes the cotton balls significantly more

hydrophobic than unpyrolyzed cotton balls, a change that was attributed to the removal of 
the polar functional groups from cellulose leaving almost pure carbon fibers, ${ }^{53,56}$ which are significantly more hydrophobic and limit the capillary action of fibers transporting the liquid agent. In order to demonstrate the hydrophobic qualities of the pyrolyzed cotton balls, a rudimentary contact angle assessment was conducted. In this study both unpyrolyzed and pyrolyzed cotton balls were placed in a dish that had a small quantity of water pipetted onto the surface. As expected, the unpyrolyzed cotton balls rapidly absorbed the water as soon as the water droplet contacted the fibers due to the hydrophilic properties of cellulose. In contrast, the pyrolyzed cotton balls did not absorb the water and instead the water droplet was suspended on the surface of the pyrolyzed cotton ball (Figure 2.9). This arrangement was observed for 5 minutes with no discernable change. While it was not possible to determine the contact angle between the water droplet and the individual pyrolyzed cotton ball fibers using this experiment, it still clearly demonstrated that the pyrolyzation process converts the cellulose fibers into nonpolar carbonized fibers which are highly hydrophobic. 


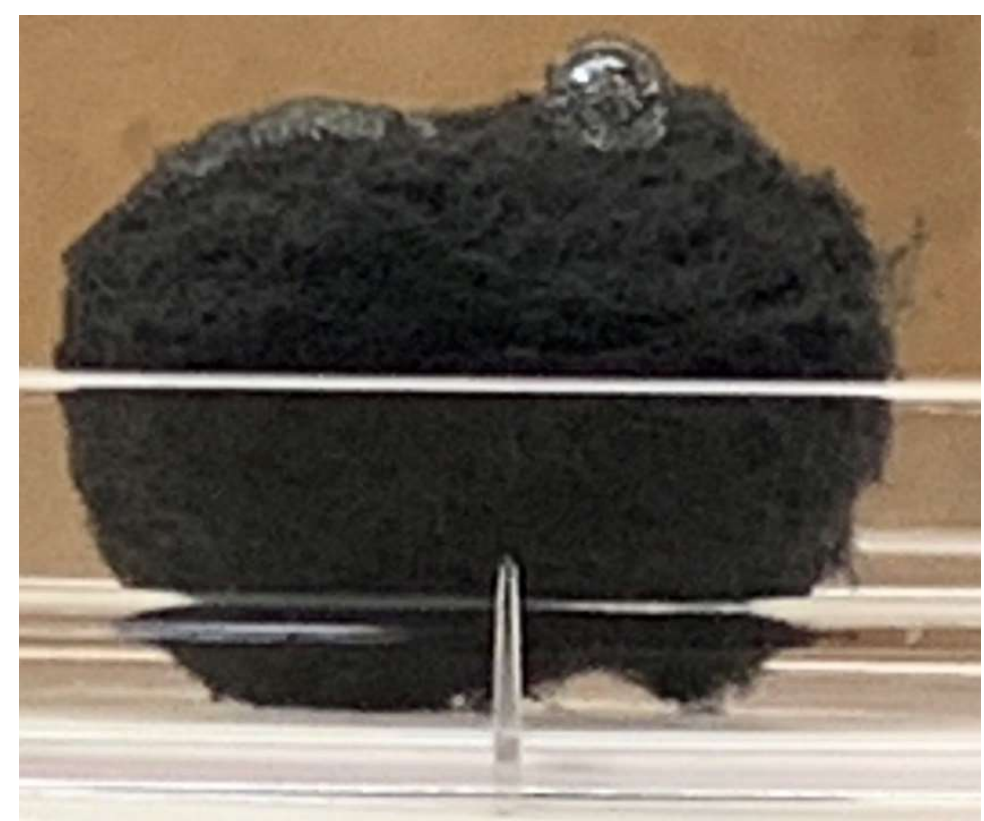

Figure 2.9: Image of pyrolyzed cotton ball with a drop of water supported on the surface due to the hydrophobic interaction between the pyrolyzed cotton ball fibers and the water droplet.

To reinforce this assessment, both unpyrolyzed and pyrolyzed cotton balls were individually suspended and slowly lowered into a watch glass filled with water. When the unpyrolyzed cotton ball fibers made contact with the surface of the water, the water began to wick up through the fibers into the bulk mass of the cotton ball. This hydrophilic interaction was strong enough that even when the cotton ball was slightly retracted from the surface of the water, the wicking continued until the cotton ball was fully saturated (Figure 2.10). Conversely, when the pyrolyzed cotton ball was lowered to the surface of the water no wicking occurred and the pyrolyzed cotton ball was suspended on the surface of the water. 


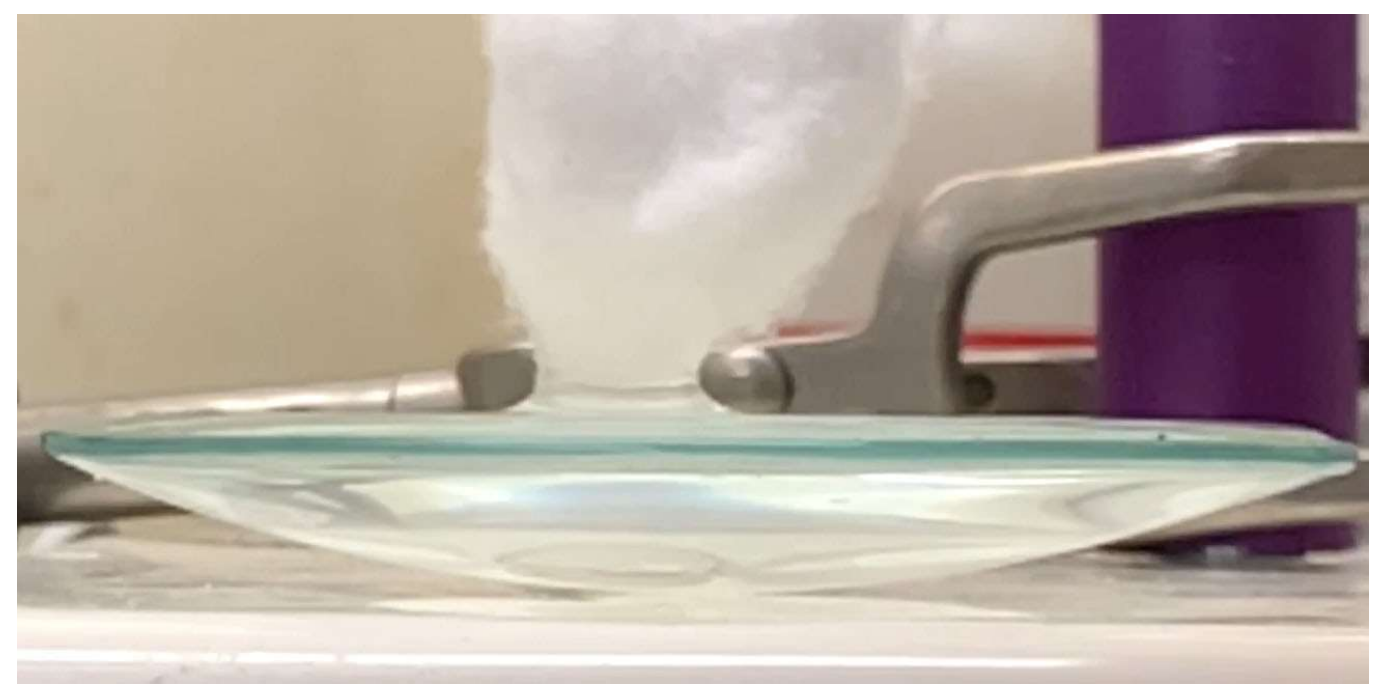

Figure 2.10: Image of the results of the hydrophilic interaction between unpyrolyzed cotton ball fibers and water as the cotton ball was slowly retracted from the surface of the water, but continued wicking the water into the bulk of the cotton ball mass

It is worth mentioning that unpyrolyzed cotton balls were saturated in just $2.5 \pm$ $0.5 \mathrm{sec}$ when placed in a pan containing the simulant (both CEES and TEP had similar results), while the PyCB required $16 \pm 5 \mathrm{sec}$ for the same level of saturation. These observations are critically important for the selected application because the unpyrolyzed cotton balls rapidly deformed when placed in the napalm mixture, while PyCB samples were able to maintain their spherical shape and hold $1.2 \pm 0.2 \mathrm{~g}$ of napalm without significant changes in morphology.

Optical imaging by the LEXT optical profiler revealed that the pyrolyzed cellulose fibers had a consistent diameter throughout the pyrolyzed cotton ball with an average size of $9.70 \pm 1.23 \mu \mathrm{m}$. By examining the height profile of the various segments, it was determined that there is significant layering within the pyrolyzed cotton ball creating voids and spaces within the structure (Figure 2.11). It is posited that these voids not only support the napalm mixture coating the pyrolyzed cotton ball but also support 
the decomposition of the chemical agent simulant as it is wicked along the pyrolyzed cellulose fibers. It is important to note that similar height profiles were observed throughout the interior and exterior of the pyrolyzed cotton balls, supporting the hypothesis of a consistent structure throughout the pyrolyzed cotton ball.
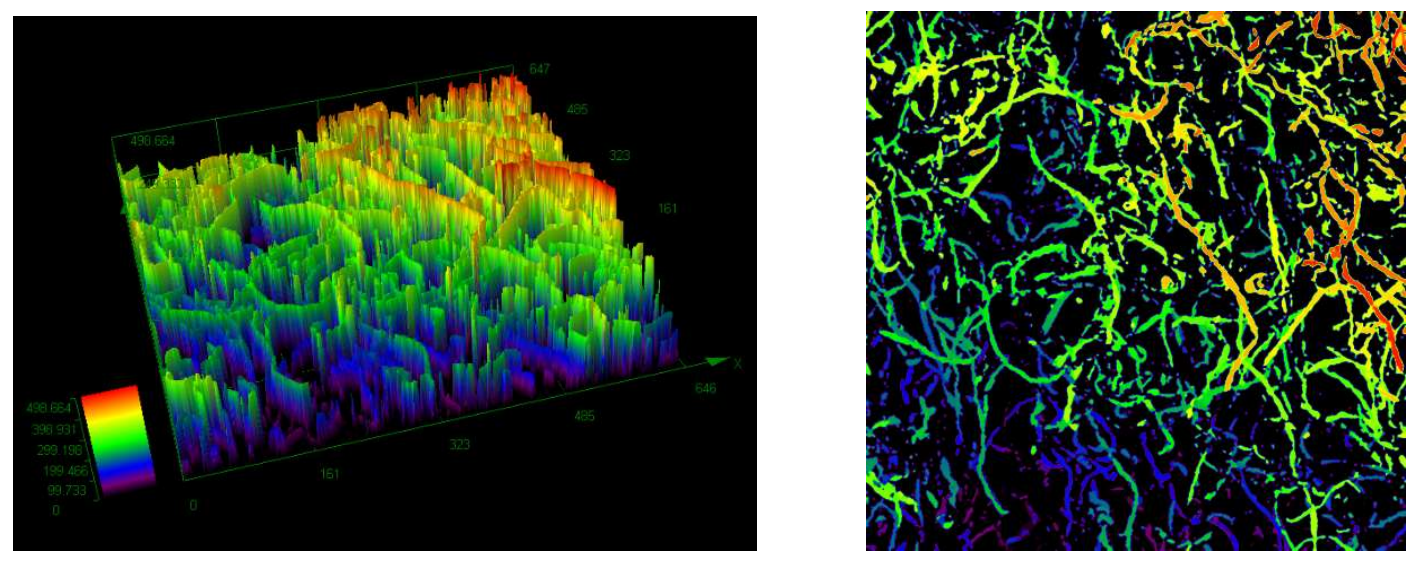

Figure 2.11: Optical non-contact roughness measurements of interior structure of pyrolyzed cotton ball: (left) three dimensional rendering of the imaged area of the pyrolyzed cotton ball displaying the varied height of the fiber in reference to the objective; (right) superimposed height image on the two dimensional image of pyrolyzed cotton ball

While there was a discrepancy between the measurement of the width of the fibers between the images obtained from the SEM and optical techniques these discrepancies were attributed to a combination of human error and slight differences in the cotton balls themselves. Ultimately the results of both analysis methods revealed that the pyrolization process resulted in a significant reduction in the size of the individual fibers as well as a more condensed structure. 


\section{Control experiments}

In order to decouple the contribution of the napalm itself from the combustion process, experiments were conducted by dripping ignited napalm directly into a sample pan containing the selected agents. While the ignited napalm without any supporting substrate was able to initiate sustained burning of CEES, the burning rate was consistently lower than the corresponding value obtained with the napalm-coated PyCB. As a representative example, the maximum burning rate of CEES achieved in an $8.5 \mathrm{~cm}$ burn pan using napalm without a substrate was $2.7 \pm 0.2 \mathrm{~g} . \mathrm{min}^{-1}$, a $14 \%$ decrease compared to the burning rate with a PyCB substrate. Additionally, the amount of time during which sustained burning of CEES occurred was reduced from $90 \pm 30 \mathrm{sec}$ (with the PyCB) to $40 \pm 20 \mathrm{sec}$ (with no substrate). Furthermore, it took an average of $60 \pm 10$ sec longer for CEES to reach a sustained burning rate when no pyrolyzed cotton ball substrate was present. It is also important to highlight that CEES is considered a flammable substance, while TEP, along with most nerve agent simulants, are considered to be non-flammable and do not ignite under most conditions. When napalm was used without a substrate there was no observable ignition of the sample as the napalm was extinguished upon contact with the liquid agent. As TEP was only ignited and reached a sustained burn rate in the presence of a PyCB substrate, these experiments illustrate the importance of the wicking material in achieving the thermal decomposition of these organophosphate containing compounds.

When the napalm was applied to a wire mesh as the substrate, it was possible to achieve some decomposition for both CEES and TEP. The maximum rate of burning 
achieved with the wire mesh and napalm above the simulant CEES was $2.19 \pm 1.25 \mathrm{~g}$. $\min ^{-1}$ which was within the standard deviation of the maximum rate of burning achieved with napalm-coated pyrolyzed cotton balls at $2.04 \pm 0.16 \mathrm{~g}$. $\mathrm{min}^{-1}$ (Table 2.1 ). The wire mesh supported napalm suspended over TEP achieved a maximum burning rate of $0.85 \pm$ 1.20 g. $\min ^{-1}, 53 \%$ of the maximum rate of burning of $1.60 \pm 0.45$ g. $\mathrm{min}^{-1}$ achieved when a pyrolyzed cotton ball wick was used. This suggests that while napalm alone is able to induce thermal decomposition of both simulants, it is necessary to have a substrate which prevents the napalm from being extinguished by the simulant. Conversely, while CEES was able to achieve burning from the ignited napalm being dripped into the simulant, this was attributed to the more volatile and flammable nature of CEES, versus the more stable organophosphate based TEP simulant. This was in agreement with previous studies, as organophosphate based compounds are commonly used as flame retardants due to the high stability of the organophosphate center. ${ }^{57,58}$

In order to demonstrate the advantages of PyCB towards the degradation of CWA simulants, thermal decomposition experiments were conducted in the presence of $\mathrm{PyCB}$ and compared to those performed with unpyrolyzed cotton balls. As unpyrolyzed cotton balls have a larger exterior surface area than the pyrolyzed cotton balls, the unpyrolyzed cotton balls were trimmed, to ensure a consistent mass of $1.2 \pm 0.1 \mathrm{~g}$ of the napalm mixture was introduced into the sample. This control of the surface area was taken due to the large decrease observed in the pyrolyzed cotton balls making it infeasible to obtain an unpyrolyzed cotton ball of the same mass as a pyrolyzed cotton ball while maintaining the same volume of napalm introduced to the system. The cotton balls were then coated 
in napalm, ignited, and placed in the sample pan. When the ignited cotton balls were placed in the pool of CEES in a $5.5 \mathrm{~cm}$ diameter sample pan a maximum burning rate of $1.21 \pm 0.37$ g. $\mathrm{min}^{-1}$ was achieved. By comparison the maximum burning rate for CEES in a $5.5 \mathrm{~cm}$ diameter pan with napalm-coated PyCBs was $2.04 \pm 0.16 \mathrm{~g}$. $\mathrm{min}^{-1}$. This represents a $40 \%$ increase in the maximum rate of burning compared to unpyrolyzed cotton balls. Interestingly, when an unpyrolyzed cotton ball was coated in napalm, ignited, and introduced to the CEES in the sample pan it was not possible to determine a rate of burning from the experimental data. It is important to note burning was observed due to the napalm-coated unpyrolyzed cotton ball, there was no measured change in the overflow rate for the 2.5-minute duration of the experiment, preventing a calculation of the mass of simulant burnt. This phenomenon was attributed to the absorption of the agent by the unpyrolyzed cellulose fibers versus the decomposition. It is important to note, that in all experiments where unpyrolyzed cotton balls were used, the unpyrolyzed cotton ball was heavily saturated with simulant, even when the experiment was allowed to burn to completion. This suggests that while the unpyrolyzed cotton ball may be able to induce thermal decomposition of some of the agent, there would be some agent remaining by physical containment in the cellulose fibers, which would require additional treatment. When pyrolyzed cotton balls were utilized there was no measurable absorption of the agent into the pyrolyzed cotton ball fibers when burning was allowed to continue until all of the sample in the pan was allowed to burn to completion.

In all trials with TEP as the simulant using unpyrolyzed cotton balls, the flame was self-extinguished within 10 seconds, thus preventing the calculation of the burning 
rate (i.e. no significant change in the rate of overflow compared to the pre-ignition rate). This suggests that the pyrolyzation process is critical for the decomposition of nerve agent simulants due to the changes in the hydrophobicity and the lack of adsorption of the liquid simulant.

When pyrolyzed cotton balls were ground into a powder and formed into a pellet with napalm as the binding agent it was possible to achieve thermal decomposition of CEES but not TEP. With CEES as the simulant in the $5.5 \mathrm{~cm}$ sample pan and the PyCB napalm pellet was introduced, the maximum burning rate achieved was $2.35 \pm 0.52 \mathrm{~g}$. $\min ^{-1}$. While this control shows that burning was achievable with the pellets, there was a relatively high degree of deviation in the maximum burning rate between the two trials conducted. This suggested that while the pellets could achieve burning of CEES the deformation of the pyrolyzed cotton balls introduces a level a variability into the preparation of the pellets that requires further optimization in order to ensure consistent results. Despite this variability, the rate of burning achieved with pyrolyzed cotton ball and napalm pellets corresponds to a slight $15 \%$ increase over the maximum rate achieved with an undeformed napalm-coated PyCBs and suggests that the pyrolyzed cellulose fibers are important in achieving greater maximum burning. Conversely, the PyCB napalm pellets were not able to induce any burning in TEP, as the flame was extinguished within 10 seconds. It is posited that the difference in chemical structure, stability, and flammability of the simulants is the main factor in this difference in achieved burning. While the precise mechanism preventing sustained ignition with the unpyrolyzed cotton balls is currently unknown, the described results present evidence that the 
structural changes in cellulose during the pyrolysis (carbonization, increase in contact angle, removal of oxygenated groups ${ }^{53}$, etc.) provide a much more efficient support to promote the combustion of both CEES and TEP. These results are summarized below in Table 2.1 and Table 2.2.

Table 2.1: Maximum rates of burning for CEES in a $5.5 \mathrm{~cm}$ sample pan with various incendiary sources

\begin{tabular}{cccccc} 
& $\begin{array}{c}\text { PyCB }+ \\
\text { Napalm }\end{array}$ & $\begin{array}{c}\text { Wire Mesh } \\
+ \text { Napalm }\end{array}$ & $\begin{array}{c}\text { PyCB }+ \\
\text { Napalm } \\
\text { Pellet }\end{array}$ & $\begin{array}{c}\text { Unpyrolyzed } \\
\text { Cotton Ball }\end{array}$ & $\begin{array}{c}\text { Unpyrolyzed } \\
\text { Cotton Ball } \\
+ \text { Napalm }\end{array}$ \\
\hline $\begin{array}{c}\text { Maximum } \\
\text { Burning Rate } \\
\left(\mathrm{g} \mathrm{min}^{-1}\right)\end{array}$ & $2.04 \pm 0.16$ & $2.19 \pm 1.25$ & $2.35 \pm 0.52$ & $1.21 \pm 0.37$ & $\begin{array}{c}\text { No } \\
\text { measured } \\
\text { burning }\end{array}$
\end{tabular}

Table 2.2: Maximum rates of burning for TEP in a $5.5 \mathrm{~cm}$ sample pan with various incendiary sources

\begin{tabular}{cccccc} 
& $\begin{array}{c}\text { PyCB }+ \\
\text { Napalm }\end{array}$ & $\begin{array}{c}\text { Wire Mesh } \\
+ \text { Napalm }\end{array}$ & $\begin{array}{c}\text { PyCB }+ \\
\text { Napalm } \\
\text { Pellet }\end{array}$ & $\begin{array}{c}\text { Unpyrolyzed } \\
\text { Cotton Ball }\end{array}$ & $\begin{array}{c}\text { Unpyrolyzed } \\
\text { Cotton Ball } \\
+ \text { Napalm }\end{array}$ \\
\hline $\begin{array}{c}\text { Maximum } \\
\text { Burning Rate } \\
\left(\mathrm{g} \mathrm{min} \text { min }^{-1}\right)\end{array}$ & $1.60 \pm 0.45$ & $0.85 \pm 1.20$ & $\begin{array}{c}\text { No } \\
\text { measured } \\
\text { burning }\end{array}$ & $\begin{array}{c}\text { No } \\
\text { measured } \\
\text { burning }\end{array}$ & $\begin{array}{c}\text { No } \\
\text { measured } \\
\text { burning }\end{array}$
\end{tabular}

It is important to note that in order to conserve the chemical warfare agent simulants used in this study for the decomposition experiments with the pyrolyzed cotton balls all control experiments were conducted in duplicate. While this resulted in higher deviation in some experiments, these controls provided a baseline for understanding the impact of both the napalm and the pyrolyzed cotton balls in achieving sustained burning for different classes of chemical warfare agent simulants. 


\section{Decomposition of 2-chloroethyl ethyl sulfide (CEES)}

While there is limited experimental research and data regarding the thermal degradation of both sulfur mustard and CEES, there is recent kinetic modeling of the reactions which predict the mechanism of decomposition. ${ }^{59}$ This model predicts under combustion or pyrolysis conditions the sulfur mustard (HD) molecule will decompose via a pericyclic pathway, cleaving the $\mathrm{C}-\mathrm{S}$ bond to produce $\mathrm{S}-\mathrm{H}$ and chlorovinyl, $\mathrm{HCl}$, or chloroethyl sulfide radicals which then decompose via a retro-ene reaction to produce vinyl chloride and thioacetaldehyde. ${ }^{59} \mathrm{~A}$ literature search revealed in studies on the decomposition of diethyl sulfide (DES), another common simulant for sulfur mustard, that under pyrolysis conditions, major products of DES combustion included ethylene, methane, ethane, and thioaldehydes. ${ }^{36,60}$ While DES lacks both terminal chlorine atoms, CEES only lacks one terminal chlorine atom when compared to the structure of sulfur mustard. Based on these slight molecular differences it follows that the resulting products of CEES decomposition would reasonably contain chloroethane and other hydrocarbons with chloride groups. Similar degradation mechanisms were found in the reaction of CEES and DES with oxidized porous carbon surfaces, supporting the hypothesis of cleaving of the C-S bond in order to both degrade the CWA and produce flammable secondary species. ${ }^{36}$ Based on the known flammability of these products and the low bond energies of the C-S bonds in both the simulants and true agent sulfur mustard, it is theorized that all of these compounds will readily decompose via thermal means. ${ }^{59}$ The literature also shows that the primary products that are released from the 
thermal decomposition of HD are carbon monoxide, carbon dioxide, water, hydrogen gas, and hydrochloric acid. ${ }^{59-61}$ Studies also concluded that while the boiling point of CEES is $156{ }^{\circ} \mathrm{C}$, in experiments where the temperature reached above $350{ }^{\circ} \mathrm{C}$ it was possible to degrade CEES, with complete degradation achieved at temperatures above $450{ }^{\circ} \mathrm{C} .{ }^{60,61}$ The temperature of the sample pan was measured with an infrared camera as experiments were conducted with napalm-coated pyrolyzed cotton balls, revealing that the temperature varied between $350-400{ }^{\circ} \mathrm{C}$. Based on the results of previous studies and the temperatures achieved with napalm-coated pyrolyzed cotton balls it was inferred that the CEES used in these thermal decomposition experiments was also degraded into the nontoxic combustion products.

To test this scenario and demonstrate the utility of the use of PyCB, experiments were performed using CEES and varying the size of the sample pan. As a summary, Figure 2.12 shows the dependence of the maximum burning rate as a function of the pan diameter. 


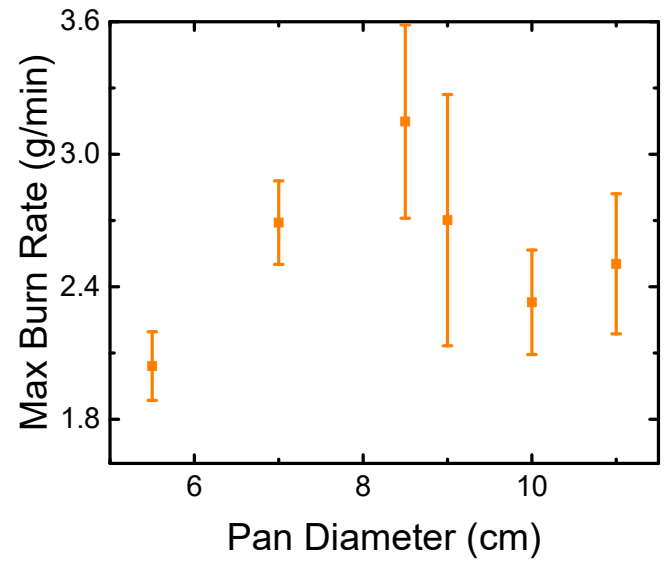

Figure 2.12: Comparison of the maximum burning rates achieved with PyCBs in CEES as a function of the diameter of the sample pan. The size of the pan does initially influence the maximum rate of burning achieved, but as the pans continue to increase in size, the rate of burning plateaus.

As observed in Figure 2.12, the maximum burning rate increases as a function of pan size from the $5.5 \mathrm{~cm}$ to the $8.5 \mathrm{~cm}$ sample pans. Then, a decrease was observed, reaching a plateau when the $10.0 \mathrm{~cm}$ diameter pans were used. This suggests there is a positive relationship between the surface area of the sample pan and the maximum rate of burning. However, if the volume of the bulk liquid agent is significantly greater than the volume of the substrate which is introduced to initiate the burning, the lower temperature of the bulk simulant will depress the rate of burning. In other words, the energy released by burning one napalm coated PyCB is not enough to cause continuously increasing rates of burning as the volume of agent in the pan increases. During the burning of CEES, the maximum burning rate was achieved after $140 \pm 20$ seconds post-ignition with burning occurring across the entire surface area of the sample pan. Due to the limited volume of the syringe pump, this maximum burning rate was sustained only for an average of $90 \pm$ 30 seconds. Across all sample pan sizes over $50 \%$ of the total burned mass was 
consumed during this short period of maximum burning, although the maximum burning time accounted for only $20 \%$ of the total burning period. Based on previous research on the reactivity of simulants as compared to the reactivity of actual warfare agents, CWAs were found to be more reactive than their respective simulants ${ }^{50}$ and a similar trend is expected for the thermal decomposition of the agents. This suggests that $\mathrm{H}$ series agents will not only be able to sustain burning, but will also achieve the maximum burning rate within 2 minutes post-ignition when a napalm coated pyrolyzed cotton ball is used as the ignition source. It should be noted that once the entire surface area of the sample pan was ignited the agent would continue to burn until all of the sample was consumed. For the purposes of this study, data taken after the volume of sample in the syringe pump was depleted was excluded.

\section{Decomposition of triethyl phosphate (TEP)}

In order to evaluate the efficacy of PyCBs to ignite other simulants, experiments were also performed using the simulant TEP while varying the size of the sample pan. As a summary, Figure 2.13 shows the dependence of the maximum burning rate as a function of the pan diameter. The maximum burning rate only showed marginal increases (within the error of the method) as the pan size increased from $5.5 \mathrm{~cm}$ to 9.0 $\mathrm{cm}$. This is a clear difference with respect to the results obtained with CEES (Figure 2.12). Additionally, TEP yielded a lower maximum burning rate (compared to CEES) across all sample pan diameters. This difference was attributed to the presence of the phosphate group which provides a high degree of stability to simulant. To illustrate the 
challenges linked to these experiments, it is important to note that organophosphates are commonly used as flame retardants due to their stability, high flash points, and resistance to burning. ${ }^{57}$ The presence of this functional group in both the simulant and the actual agents contributes to an increased flash point, making these nerve agents significantly more resistant towards combustion than the $\mathrm{H}$ series agents. Interestingly, the smallest pan $(5.5 \mathrm{~cm}$ diameter $)$ exhibited a much more consistent maximum burning rate across all repetitions $(\mathrm{n}=3)$.

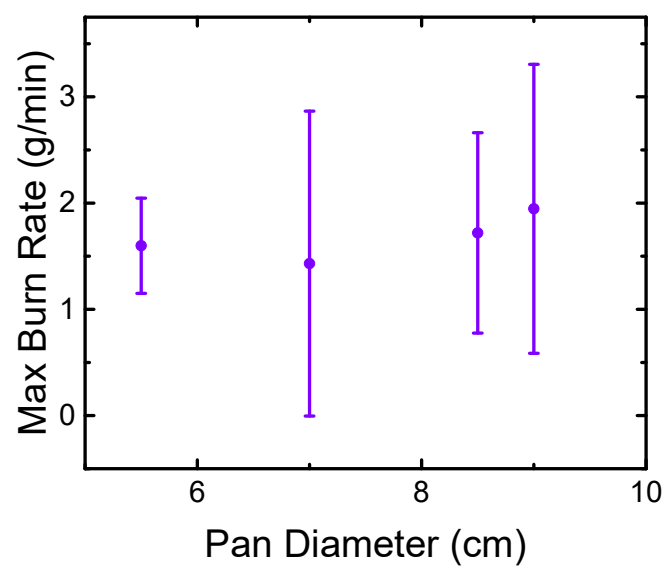

Figure 2.13: Comparison of maximum burning rates achieved with PyCBs in TEP as a function of the diameter of the sample pan. The size of the pan appears to have minimal impact on the rate of burning achieved when TEP is used.

During the burning experiments it was observed that a white layer of vapor was produced above the liquid agent, and in the smaller diameter pans this vapor would ignite, resulting in sustained burning across the total surface area of the sample pans. The formation of this vapor, which was more pronounced in smaller pans, was attributed to a pericyclic reaction that results in the fragmentation TEP, a retro-ene reaction as presented in Figure 2.14. ${ }^{62,63}$ In this reaction, the energy from the burning napalm causes the 
pericyclic reaction, resulting in the expulsion of highly flammable ethylene. The ethylene produced from this reaction is posited as the major fuel component in the decomposition of TEP and is continuously released by the proposed mechanism below, eventually producing the less toxic phosphoric acid.

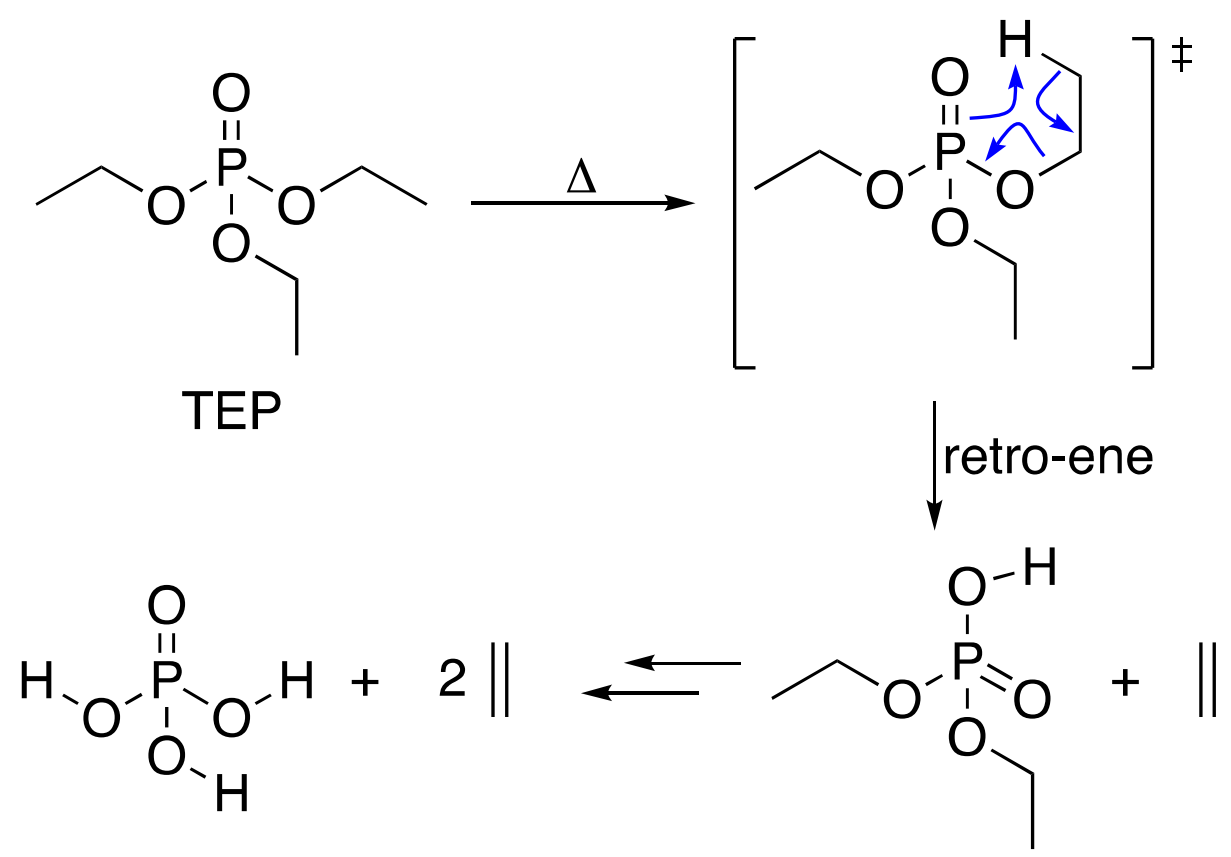

Figure 2.14: Proposed reaction mechanism for TEP generating flammable ethylene and the less toxic by-product, phosphoric acid. This reaction requires the high temperatures from the burning of napalm in order to liberate the ethylene and to proliferate the reaction until all ethyl groups are removed, leaving only phosphoric acid. 


\section{CHAPTER THREE}

\section{CONCLUSIONS AND FUTURE RESEARCH}

\section{Conclusions of pyrolyzed cotton ball experiments}

The method shown in this paper is the first known study where a carbon-based material is used as a wick to thermally decompose different classes of chemical warfare agents under ambient conditions. Not only are PyCBs able to serve as effective substrates for the delivery of relatively large volumes of an incendiary agent relative to the size and mass of the substrate itself, but they also increase the rate of decomposition when compared to the incendiary agent alone. Furthermore, PyCBs enable the rapid decomposition of bulk volumes of agents with very limited by-products. While further studies are required to confirm the exact composition of the gases and fumes produced by the thermal degradation of these compounds, the ability to rapidly decompose large volumes of these deadly substances is a significant improvement in the efforts to eliminate the stockpiles of these weapons at low cost. This study also presents a fielddeployable method for rapid and simple decomposition of a broad range of chemical warfare agents with limited sample preparation or specialized training for the end user.

In general, the structural changes induced by the pyrolysis of the cotton balls enhanced the ability of the napalm mixture to continuously combust when introduced into the pan containing the CWA simulants. This was due to its ability to increase the temperature of the surrounding agent to the point of ignition and allow the agent itself to fuel the combustion reaction. 


\section{Future research}

While pyrolyzed cotton balls present a novel method for achieving thermal decomposition of chemical warfare agents and their simulants, further research is required into implementing this method into bulk decomposition. It was shown that there is a positive relationship between the size of the sample pan used to hold the agent and the achievable rate of burning, however this is limited. Further research would examine if increasing the number of pyrolyzed cotton balls would further increase the rates of burning. This would also reveal if there is a positive relationship between the volume of liquid agent present and the volume of the pyrolyzed cotton balls present. Additional research is needed in the delivery method for presenting the ignited pyrolyzed cotton balls coated in napalm to the agent. While the method used in this study was adequate for laboratory scale experiments, bulk or stockpile-scale experiments would require different methods primarily due to safety concerns of these large volumes of toxic substances.

Further research is also required into the analyzing the products, especially in the gaseous state, of the thermal decomposition. While the scope of this study was restricted to developing a cheaper and easier method towards igniting CWA and their simulants, the hazard of releasing toxic substances cannot be ignored, especially when larger scales are being examined. As shown by the United States Army's previous attempts to utilize incendiary methods to decompose its chemical weapons stockpile in the 1980 s, there are significant regulations and political challenges to any method which releases potentially

toxic by-products into the atmosphere. ${ }^{25,64,65}$ Further examination of the vapors and by- 
products of thermal decomposition of both simulants and CWA should be examined via headspace analysis of the gaseous products before the presented method is applied to larger scale experiments to confirm that the target substances are thoroughly degraded into non-toxic byproducts. 


\section{REFERENCES}

(1) Giannakoudakis, D. A.; Bandosz, T. J. Detoxification of Chemical Warfare Agents; Springer, Cham, 2018.

(2) Liang, H.; Yao, A.; Jiao, X.; Li, C.; Chen, D. Fast and Sustained Degradation of Chemical Warfare Agent Simulants Using Flexible Self-Supported Metal-Organic Framework Filters. ACS Appl. Mater. Interfaces 2018, 10 (24), 20396-20403. https://doi.org/10.1021/acsami.8b02886.

(3) Lee, D. T.; Zhao, J.; Oldham, C. J.; Peterson, G. W.; Parsons, G. N. UiO-66-NH2 Metal-Organic Framework (MOF) Nucleation on $\mathrm{TiO} 2, \mathrm{ZnO}$, and Al2O3 Atomic Layer Deposition-Treated Polymer Fibers: Role of Metal Oxide on MOF Growth and Catalytic Hydrolysis of Chemical Warfare Agent Simulants. ACS Appl. Mater. Interfaces 2017, 9 (51), 44847-44855. https://doi.org/10.1021/acsami.7b15397.

(4) Borak, J.; Sidell, F. R. Agents of Chemical Warfare: Sulfur Mustard. Ann. Emerg. Med. 1992, 21 (3), 303-308. https://doi.org/10.1016/S0196-0644(05)80892-3.

(5) Blewett, W. Tactical Weapons: Is Mustard Still King? NBC Def. Technol. Int. 1986, 1, 64-66.

(6) Gilman, A.; Phillips, F. S. The Biological Actions and Therapeutic Applications of the B-Chloroethyl Amines and Sulfides. Science (80-. ). 1946, 103, 409-415.

(7) Dunn, P. The Chemical War: Iran Revisited 1986. NBC Def. Technol. Int. 1986, 1, 32-39.

(8) McNaugher, T. L. Ballistic Missles and Chemical Weapons: The Legacy of the Iran-Iraq War. Int. Secur. 1990, 15 (2), 5-34.

(9) Chemical Agent Data Sheets Vol 1; 1974.

(10) Munro, N. B.; Ambrose, K. R.; Watson, A. P. Toxicity of the Organophosphate Chemical Warfare Agents GA, GB, and VX: Implications for Public Protection. Environ. Health Perspect. 1994, 102 (1), 18-38.

(11) Kloske, M.; Witkiewicz, Z. Novichoks - The A Group of Organophosphorus Chemical Warfare Agents. Chemosphere 2019, 221, 672-682. https://doi.org/10.1016/j.chemosphere.2019.01.054.

(12) Grob, D.; Harver, A. M. The Effects and Treatment of Nerve Gas Poisoning. Am. J. Med. 1953, 14 (1), 52-63.

(13) Bowers, M. B.; Goodman, E.; Sim, V. M. Some Behavioral Changes in Man Following Anticholinesterase Administration. J. Nerv. Ment. Dis. 1964, 138 (4), 383-389.

(14) Chemical Agent Data Sheet; Aberdeen Proving Ground, MD, 1974.

(15) Munro, N. B.; Watson, A. P.; Ambrose, K. R.; Griffin, G. D. Treating Exposure to Chemical Warfare Agents: Implications for Health Care Providers and Community Emergency Planning. Environ. Health Perspect. 1990, 89 (May), 205-215. https://doi.org/10.1289/ehp.9089205.

(16) Love, A. H.; Vance, A. L.; Reynolds, J. G.; Davisson, M. L. Investigating the Affinities and Persistence of VX Nerve Agent in Environmental Matrices. Chemosphere 2004, 57 (10), 1257-1264. https://doi.org/10.1016/j.chemosphere.2004.08.041. 
(17) Sidell, F. R.; Groff, W. A. The Reactivatibility of Cholinesterase Inhibited by VX and Sarin in Man. Toxicol. Appl. Pharmacol. 1974, 27 (2), 241-252.

(18) Schneider, T.; Lütkefend, T. Nowhere to Hide: The Logic of Chemical Weapons Use in Syria. Glob. Public Policy Inst. 2019.

(19) Yang, Y. C.; Baker, J. A.; Ward, R. Decontamination of Chemical Warfare Agents. Chem. Rev. 1992, 92, 1729-1743. https://doi.org/10.1002/9783527621620.ch7.

(20) Yang, Y. C. Chemical Detoxification of Nerve Agent VX. Acc. Chem. Res. 1999, 32 (2), 109-115. https://doi.org/10.1021/ar970154s.

(21) Jõul, P.; Vaher, M.; Kuhtinskaja, M. Evaluation of Carbon Aerogel-Based SolidPhase Extraction Sorbent for the Analysis of Sulfur Mustard Degradation Products in Environmental Water Samples. Chemosphere 2018, 198, 460-468. https://doi.org/10.1016/j.chemosphere.2018.01.157.

(22) Petrea, N.; Petre, R.; Epure, G.; Şomoghi, V.; TĂnase, L. C.; Teodorescu, C. M.; Neaţu, Ş. The Combined Action of Methanolysis and Heterogeneous Photocatalysis in the Decomposition of Chemical Warfare Agents. Chem. Commun. 2016, 52 (88), 12956-12959. https://doi.org/10.1039/c6cc07551g.

(23) Kim, M. K.; Kim, S. H.; Park, M.; Ryu, S. G.; Jung, H. Degradation of Chemical Warfare Agents over Cotton Fabric Functionalized with UiO-66-NH 2. RSC Adv. 2018, 8 (72), 41633-41638. https://doi.org/10.1039/c8ra06805d.

(24) U.S. Congress Office of Technology Assessment. Disposal of Chemical Weapons : Alternative Technologies-Background Paper OTA-BP-O-95; Washington, DC, 1992.

(25) Flamm, K. J.; Kwan, Q.; McNulty, W. B. Chemical Agent and Munition Disposal: Summary of the U.S. Army's Experience; Aberdeen Proving Ground, MD, 1987.

(26) Nath, I.; Chakraborty, J.; Verpoort, F. Metal Organic Frameworks Mimicking Natural Enzymes: A Structural and Functional Analogy. Chem. Soc. Rev. 2016, 45 (15), 4127-4170. https://doi.org/10.1039/c6cs00047a.

(27) Bobbitt, N. S.; Mendonca, M. L.; Howarth, A. J.; Islamoglu, T.; Hupp, J. T.; Farha, O. K.; Snurr, R. Q. Metal-Organic Frameworks for the Removal of Toxic Industrial Chemicals and Chemical Warfare Agents. Chem. Soc. Rev. 2017, 46 (11), 3357-3385. https://doi.org/10.1039/c7cs00108h.

(28) Zhao, J.; Lee, D. T.; Yaga, R. W.; Hall, M. G.; Barton, H. F.; Woodward, I. R.; Oldham, C. J.; Walls, H. J.; Peterson, G. W.; Parsons, G. N. Ultra-Fast Degradation of Chemical Warfare Agents Using MOF-Nanofiber Kebabs. Angew. Chemie - Int. Ed. 2016, 55 (42), 13224-13228. https://doi.org/10.1002/anie.201606656.

(29) Furukawa, H.; Cordova, K. E.; O'Keeffe, M.; Yaghi, O. M. The Chemistry and Applications of Metal-Organic Frameworks. Science (80-. ). 2013, 341 (6149). https://doi.org/10.1126/science.1230444.

(30) Long, J. R.; Yaghi, O. M. The Pervasive Chemistry of Metal-Organic Frameworks. Chem. Soc. Rev. 2009, 38 (5), 1213-1214. https://doi.org/10.1039/b903811f. 
(31) Mondloch, J. E.; Katz, M. J.; Isley, W. C.; Ghosh, P.; Liao, P.; Bury, W.; Wagner, G. W.; Hall, M. G.; Decoste, J. B.; Peterson, G. W.; et al. Destruction of Chemical Warfare Agents Using Metal-Organic Frameworks. Nat. Mater. 2015, 14 (5), 512516. https://doi.org/10.1038/nmat4238.

(32) Katz, M. J.; Mondloch, J. E.; Totten, R. K.; Park, J. K.; Nguyen, S. T.; Farha, O. K.; Hupp, J. T. Simple and Compelling Biomimetic Metal-Organic Framework Catalyst for the Degradation of Nerve Agent Simulants. Angew. Chemie - Int. Ed. 2014, 53 (2), 497-501. https://doi.org/10.1002/anie.201307520.

(33) Moon, S. Y.; Proussaloglou, E.; Peterson, G. W.; DeCoste, J. B.; Hall, M. G.; Howarth, A. J.; Hupp, J. T.; Farha, O. K. Detoxification of Chemical Warfare Agents Using a Zr6-Based Metal-Organic Framework/Polymer Mixture. Chem. A Eur. J. 2016, 22 (42), 14864-14868. https://doi.org/10.1002/chem.201603976.

(34) Wang, S.; Bromberg, L.; Schreuder-Gibson, H.; Hatton, T. A. Organophophorous Ester Degradation by Chromium(III) Terephthalate Metal-Organic Framework (MIL-101) Chelated to N, N-Dimethylaminopyridine and Related Aminopyridines. ACS Appl. Mater. Interfaces 2013, 5 (4), 1269-1278. https://doi.org/10.1021/am302359b.

(35) Lõpez-Maya, E.; Montoro, C.; Rodríguez-Albelo, L. M.; Aznar Cervantes, S. D.; Lozano-Pérez, A. A.; Cenís, J. L.; Barea, E.; Navarro, J. A. R. Textile/MetalOrganic-Framework Composites as Self-Detoxifying Filters for Chemical-Warfare Agents. Angew. Chemie - Int. Ed. 2015, 54 (23), 6790-6794.

https://doi.org/10.1002/anie.201502094.

(36) Florent, M.; Giannakoudakis, D. A.; Bandosz, T. J. Mustard Gas Surrogate Interactions with Modified Porous Carbon Fabrics: Effect of Oxidative Treatment. Langmuir 2017, 33 (42), 11475-11483. https://doi.org/10.1021/acs.langmuir.7b02047.

(37) Picard, B.; Chataigner, I.; Maddaluno, J.; Legros, J. Introduction to Chemical Warfare Agents, Relevant Simulants and Modern Neutralisation Methods. Org. Biomol. Chem. 2019, 17 (27), 6528-6537. https://doi.org/10.1039/c9ob00802k.

(38) Singh, B.; Prasad, G. K.; Pandey, K. S.; Danikhel, R. K.; Vijayaraghavan, R. Decontamination of Chemical Warfare Agents. Def. Sci. J. 2010, 60 (4), 428-441. https://doi.org/10.1016/B978-012374484-5.00071-7.

(39) Zhang, W.; Sun, H.; Chen, W.; Zhang, Y.; Wang, F.; Tang, S.; Zhang, J.; Wang, H.; Wang, R. Mechanistic and Kinetic Study on the Reaction of Ozone and Trans2-Chlorovinyldichloroarsine. Chemosphere 2016, 150, 329-340. https://doi.org/10.1016/j.chemosphere.2016.01.115.

(40) Sharma, V. K.; Luther, G. W.; Millero, F. J. Mechanisms of Oxidation of Organosulfur Compounds by Ferrate(VI). Chemosphere 2011, 82 (8), 1083-1089. https://doi.org/10.1016/j.chemosphere.2010.12.053.

(41) Li, Y.; Gao, Q.; Zhou, Y.; Zhang, L.; Zhong, Y.; Ying, Y.; Zhang, M.; Liu, Y.; Wang, Y. Significant Enhancement in Hydrolytic Degradation of Sulfur Mustard Promoted by Silver Nanoparticles in the Ag NPs@HKUST-1 Composites. J. Hazard. Mater. 2018, 358 (January), 113-121. https://doi.org/10.1016/j.jhazmat.2018.06.043. 
(42) Verma, A. K.; Srivastava, A. K.; Singh, B.; Shah, D.; Shrivastava, S.; Shinde, C. K. P. Alumina-Supported Oxime for the Degradation of Sarin and Diethylchlorophosphate. Chemosphere 2013, 90 (8), 2254-2260. https://doi.org/10.1016/j.chemosphere.2012.10.011.

(43) Veriansyah, B.; Kim, J. D.; Lee, J. C. Destruction of Chemical Agent Simulants in a Supercritical Water Oxidation Bench-Scale Reactor. J. Hazard. Mater. 2007, 147 (1-2), 8-14. https://doi.org/10.1016/j.jhazmat.2006.12.040.

(44) Veriansyah, B.; Kim, J. D.; Lee, J. C.; Lee, Y. W. OPA Oxidation Rates in Supercritical Water. J. Hazard. Mater. 2005, 124 (1-3), 119-124. https://doi.org/10.1016/j.jhazmat.2005.04.019.

(45) Yesodharan, S. Supercritical Water Oxidation: An Environmentally Safe Method for the Disposal of Organic Wastes. Curr. Sci. 2002, 82 (9), 1112-1122.

(46) Sides, G. D.; Manier, M. L. Pyrolysis of Bis(2-Chloroethyl) Sulfide (HD); 1985.

(47) Sides, G.; Manier, M. L.; Spafford, R. B. The Pyrolysis of VX GB HD Trapped in Stainless Steel Reaction Vessels; 1986.

(48) Zavitsanos, P.; Rozanski, A.; Files, C.; Stotz, N. Destruction of CWA by Wicking Compound and High Temperature Incendiary. 9610468 B2, 2017.

(49) Kim, K.; Tsay, O. G.; Atwood, D. A.; Churchill, D. G. Destruction and Detection of Chemical Warfare Agents. Chem. Rev. 2011, 111, 5345-5403. https://doi.org/10.1021/acs.chemrev.5b00402.

(50) Kumar, V.; Raviraju, G.; Rana, H.; Rao, V. K.; Gupta, A. K. Highly Selective and Sensitive Chromogenic Detection of Nerve Agents (Sarin, Tabun and VX): A Multianalyte Detection Approach. Chem. Commun. 2017, 53 (96), 12954-12957. https://doi.org/10.1039/c7cc07823d.

(51) Bartelt-Hunt, S. L.; Knappe, D. R. U.; Barlaz, M. A. A Review of Chemical Warfare Agent Simulants for the Study of Environmental Behavior. Crit. Rev. Environ. Sci. Technol. 2008, 38 (2), 112-136. https://doi.org/10.1080/10643380701643650.

(52) Wallace, K. J.; Morey, J.; Lynch, V. M.; Anslyn, E. V. Colorimetric Detection of Chemical Warfare Simulants. New J. Chem. 2005, 29 (11), 1469-1474. https://doi.org/10.1039/b506100h.

(53) Benavidez, T. E.; Martinez-Duarte, R.; Garcia, C. D. Analytical Methodologies Using Carbon Substrates Developed by Pyrolysis. Anal. Methods 2016, 8 (21), 4163-4176. https://doi.org/10.1039/c6ay00293e.

(54) Özdemir, M.; Bolgaz, T.; Saka, C.; Şahin, Ö. Preparation and Characterization of Activated Carbon from Cotton Stalks in a Two-Stage Process. J. Anal. Appl. Pyrolysis 2011, 92 (1), 171-175. https://doi.org/10.1016/j.jaap.2011.05.010.

(55) Long, D.; Zhang, R.; Qiao, W.; Zhang, L.; Liang, X.; Ling, L. Biomolecular Adsorption Behavior on Spherical Carbon Aerogels with Various Mesopore Sizes. J. Colloid Interface Sci. 2009, 331 (1), 40-46. https://doi.org/10.1016/j.jcis.2008.11.026. 
(56) Giuliani, J. G.; Benavidez, T. E.; Duran, G. M.; Vinogradova, E.; Rios, A.; Garcia, C. D. Development and Characterization of Carbon Based Electrodes from Pyrolyzed Paper for Biosensing Applications. J. Electroanal. Chem. 2016, 765, 815. https://doi.org/10.1016/j.jelechem.2015.07.055.

(57) Deng, J.; Zhang, P.; Jin, T.; Zhou, H.; Cheng, J. Graphene Oxide/ $\beta$-Cyclodextrin Composite as Fiber Coating for High Efficiency Headspace Solid Phase Microextraction of Organophosphate Ester Flame Retardants in Environmental Water. RSC Adv. 2017, 7 (86), 54475-54484. https://doi.org/10.1039/c7ra07903f.

(58) Yuan, X.; Lacorte, S.; Cristale, J.; Dantas, R. F.; Sans, C.; Esplugas, S.; Qiang, Z. Removal of Organophosphate Esters from Municipal Secondary Effluent by Ozone and UV/H2O2 Treatments. Sep. Purif. Technol. 2015, 156, 1028-1034. https://doi.org/10.1016/j.seppur.2015.09.052.

(59) Sirjean, B.; Lizardo-Huerta, J. C.; Verdier, L.; Fournet, R.; Glaude, P. A. Kinetic Modeling of the Thermal Destruction of Mustard Gas. Proc. Combust. Inst. 2017, 36 (1), 499-506. https://doi.org/10.1016/j.proci.2016.05.016.

(60) Zheng, X.; Fisher, E. M.; Gouldin, F. C.; Zhu, L.; Bozzelli, J. W. Experimental and Computational Study of Diethyl Sulfide Pyrolysis and Mechanism. Proc. Combust. Inst. 2009, 32, 469-476. https://doi.org/10.1016/j.proci.2008.06.176.

(61) Wagner, G. W.; Maciver, B. K.; Rohrbaugh, D. K.; Yang, Y. C. Thermal Degradation of Bis (2-Chloroethyl) Sulfide (Mustard Gas). Phosphorus, Sulfur Silicon Relat. Elem. 1999, 152, 65-76. https://doi.org/10.1080/10426509908031618.

(62) Paderes, G. D.; Jorgensen, W. L. Computer-Assisted Mechanistic Evaluation of Organic Reactions. 20. Ene and Retro-Ene Chemistry. J. Org. Chem. 1992, 57 (6), 1904-1916. https://doi.org/10.1021/jo00032a054.

(63) Bruffaerts, J.; Vasseur, A.; Marek, I. Alkene-Zipper Catalyzed Selective and Remote Retro-Ene Reaction of Alkenyl Cyclopropylcarbinol. Adv. Synth. Catal. 2018, 360 (7), 1389-1396. https://doi.org/10.1002/adsc.201701481.

(64) Carnes, S. A. Disposing of the US Chemical Weapons Stockpile: An Approaching Reality. J. Am. Med. Assoc. 1989, 262 (5), 653-659.

(65) Greenberg, M. R. Public Health, Law, and Local Control: Destruction of the US Chemical Weapons Stockpile. Am. J. Public Health 2003, 93 (8), 1222-1226. https://doi.org/10.2105/AJPH.93.8.1222. 